\title{
First direct detection of a polarized companion outside a resolved circumbinary disk around CS Chamaeleonis ${ }^{\star} \star \star$
}

\author{
C. Ginski ${ }^{1,2}$, M. Benisty ${ }^{3,4}$, R. G. van Holstein ${ }^{1}$, A. Juhász ${ }^{5}$, T. O. B. Schmidt ${ }^{6}$, G. Chauvin ${ }^{3,4}$, J. de Boer ${ }^{1}$, \\ M. Wilby ${ }^{1}$, C. F. Manara ${ }^{7}$, P. Delorme ${ }^{4}$, F. Ménard ${ }^{4}$, P. Pinilla ${ }^{8}$, T. Birnstiel ${ }^{9}$, M. Flock ${ }^{10}$, C. Keller ${ }^{1}$, \\ M. Kenworthy ${ }^{1}$, J. Milli ${ }^{4,11}$, J. Olofsson ${ }^{12,13}$, L. Pérez ${ }^{14}$, F. Snik ${ }^{1}$, and N. Vogt ${ }^{12}$ \\ ${ }^{1}$ Leiden Observatory, Leiden University, PO Box 9513, 2300 RA Leiden, The Netherlands \\ e-mail: ginski@strw.leidenuniv.nl \\ 2 Anton Pannekoek Institute for Astronomy, University of Amsterdam, Science Park 904, 1098 XH Amsterdam, The Netherlands \\ 3 Unidad Mixta Internacional Franco-Chilena de Astronomía, CNRS/INSU UMI 3386 and Departamento de Astronomía, \\ Universidad de Chile, Casilla 36-D, Santiago, Chile \\ ${ }^{4}$ Université Grenoble Alpes, CNRS, IPAG, 38000 Grenoble, France \\ 5 Institute of Astronomy, University of Cambridge, Madingley Road, Cambridge CB3 0HA, UK \\ ${ }^{6}$ LESIA, Observatoire de Paris, PSL Research University, CNRS, Sorbonne Universités, UPMC Univ. Paris 06, Univ. Paris Diderot, \\ Sorbonne Paris Cité, 5 place Jules Janssen, 92195 Meudon, France \\ 7 Scientific Support Office, Directorate of Science, European Space Research and Technology Centre (ESA/ESTEC), Keplerlaan 1, \\ 2201 AZ Noordwijk, The Netherlands \\ 8 Department of Astronomy/Steward Observatory, The University of Arizona, 933 North Cherry Avenue, Tucson, AZ 85721, USA \\ 9 University Observatory, Faculty of Physics, Ludwig-Maximilians-Universität München, Scheinerstr. 1, 81679 Munich, Germany \\ 10 Max-Planck-Institut für Astronomie, Königstuhl 17, 69117 Heidelberg, Germany \\ 11 European Southern Observatory (ESO), Alonso de Córdova 3107, Vitacura, Casilla 19001, Santiago, Chile \\ 12 Instituto de Física y Astronomía, Facultad de Ciencias, Universidad de Valparaíso, Av. Gran Bretaña 1111, Valparaíso, Chile \\ 13 Núcleo Milenio Formación Planetaria - NPF, Universidad de Valparaíso, Av. Gran Bretaña 1111, Valparaíso, Chile \\ ${ }^{14}$ Universidad de Chile, Departamento de Astronomía, Camino El Observatorio 1515, Las Condes, Santiago, Chile
}

Received 4 December 2017 / Accepted 27 April 2018

\begin{abstract}
Aims. To understand planet formation it is necessary to study the birth environment of planetary systems. Resolved imaging of young planet forming disks allows us to study this environment in great detail and find signs of planet-disk interaction and disk evolution. In the present study we aim to investigate the circumstellar environment of the spectroscopic binary T Tauri star CS Cha. From unresolved mid- to far-infrared photometry it is predicted that CS Cha hosts a disk with a large cavity. In addition, spectral energy distribution modeling suggests significant dust settling, pointing toward an evolved disk that may show signs of ongoing or completed planet formation. Methods. We observed CS Cha with the high contrast imager SPHERE at the ESO Very Large Telescope (VLT) in polarimetric differential imaging mode to resolve the circumbinary disk in near-infrared scattered light. These observations were followed up by VLT/NACO $L$-band observations and complemented by archival VLT/NACO $K$-band and Hubble Space Telescope WFPC2 I-band data.

Results. We resolve the compact circumbinary disk around CS Cha for the first time in scattered light. We find a smooth, low inclination disk with an outer radius of $\sim 55 \mathrm{au}$ (at $165 \mathrm{pc}$ ). We do not detect the inner cavity but find an upper limit for the cavity size of $\sim 15$ au. Furthermore, we find a faint comoving companion with a projected separation of 210 au from the central binary outside of the circumbinary disk. The companion is detected in polarized light and shows an extreme degree of polarization $(13.7 \pm 0.4 \%$ in the $J$ band). The $J$ - and $H$-band magnitudes of the companion are compatible with masses of a few $M_{\text {Jup }}$. However, $K$-, $L$-, and $I$-band data draw this conclusion into question. We explore with radiative transfer modeling whether an unresolved circum-companion disk can be responsible for the high polarization and complex photometry. We find that the set of observations is best explained by a heavily extincted low-mass ( $\sim 20 M_{\text {Jup }}$ ) brown dwarf or high-mass planet with an unresolved disk and dust envelope.
\end{abstract}

Key words. stars: individual: CS Cha - protoplanetary disks - planet-disk interactions - techniques: polarimetric

\section{Introduction}

In the past few years high contrast and high resolution observations across a large wavelength range have revealed a variety of distinct features in planet forming disks. Multiple ringed

\footnotetext{
^ Based on observations performed with VLT/SPHERE under program ID 098.C-0760(B) and 099.C-0891(B) and VLT/NACO under program ID 298.C-5054(B) and 076.C-0292(A)

$\star \star$ The reduced images are only available at the CDS via anonymous ftp to cdsarc.u-strasbg.fr (130.79.128.5) or via http://cdsarc.u-strasbg.fr/viz-bin/qcat?]/A+A/616/A79
}

systems were uncovered such as HL Tau (ALMA Partnership et al. 2015), HD 97048 (Walsh et al. 2016; Ginski et al. 2016; van der Plas et al. 2017), or TW Hya (Andrews et al. 2016; van Boekel et al. 2017). Other systems such as MWC 758 (Grady et al. 2013; Benisty et al. 2015), HD 100453 (Wagner et al. 2015; Benisty et al. 2017), or Elias 2-27 (Pérez et al. 2016) show huge spiral arms or variable shadows (HD 135344 B, Stolker et al. 2017b). It is still unclear whether these features in general or in part are linked to ongoing planet formation or rather to other processes within the disks. In addition to ever more detailed images of circumstellar disks, a growing number of 
giant planets at wide orbital separations (typically $>100 \mathrm{au}$ ) are discovered (e.g., HD 106906 b, Bailey et al. 2014; HD 203030 b, Metchev \& Hillenbrand 2006; CVSO 30 c, Schmidt et al. 2016). These objects are of particular interest to understand planet formation mechanisms, since they are the youngest planets that we have discovered and we can study their atmospheres in great detail via resolved spectroscopy. Yet these objects are also particularly puzzling because typical planet formation mechanisms such as core accretion should take much longer than $100 \mathrm{Myr}$ at these distances (Pollack et al. 1996), while the typical dissipation timescale of gas-rich disks is at least an order of magnitude shorter (Haisch et al. 2001). Clearly, detailed characterization of other, younger, systems is required to refine the current paradigm and to understand whether the observed disk structures are linked to planet formation. In this work we concentrate on a previously unresolved disk around a nearby $\mathrm{T}$ Tau object.

CSCha is a young ( $2 \pm 2 \mathrm{Myr}$, Luhman et al. 2008) classical T Tauri object of spectral type K2Ve (Appenzeller 1977; Manara et al. 2014), located in the Chamaeleon I association at a distance of $165 \pm 30 \mathrm{pc}$ (combined estimate from Whittet et al. 1997; Bertout et al. 1999 following Schmidt et al. 2008) ${ }^{1}$. Guenther et al. (2007) found that CS Cha is likely a single lined spectroscopic binary with a minimum mass of the secondary component of $0.1 M_{\odot}$ and a minimum orbital period of $2482 \mathrm{~d}$ ( $\sim 4 \mathrm{au}$ semimajor axis, assuming a system mass of $\left.1 M_{\odot}\right)$. In a later study by Nguyen et al. (2012) the binary nature of CSCha was confirmed. These authors were able to fit the broadened spectral lines with two Gaussian profiles, making the system potentially a double lined spectroscopic binary. They found a flux ratio of the two components of 1.0 0.4.

CS Cha is well known to feature a large infrared excess in its spectral energy distribution (SED) with a pronounced dip at $10 \mu \mathrm{m}$ (see, e.g., Gauvin \& Strom 1992). The lack of emission at this wavelength regime was attributed to a large cavity by several studies (Gauvin \& Strom 1992; Espaillat et al. 2007, 2011; Kim et al. 2009; Ribas et al. 2016), indicating that the system might be in a transition stage from a young gas-rich disk to a debris disk. The radius of the cavity has been a subject of intense modeling using unresolved photometric measurements. Espaillat et al. $(2007,2011)$ found rather large cavity radii between $38 \mathrm{au}$ and $43 \mathrm{au}$, while a more recent study by Ribas et al. (2016) based on Herschel data has estimated a smaller radius of $18_{-5}^{+6}$ au. The most likely explanation is that the disk cavity is caused entirely by the stellar binary companion, since the cavity size is within a factor of a few of the binary semimajor axis. ALMA band 3 observations by Dunham et al. (2016) did not resolve the disk with a beam size of $2.7 \times 1.9$ arcsec, limiting the outer extent of the disk to radii smaller than 169 au for the population of millimeter-sized dust grains.

Radiative transfer modeling of the unresolved photometry by Espaillat et al. (2007) suggested that significant dust settling and large dust grains $(5 \mu \mathrm{m})$ are needed to fit the SED in the farinfrared and millimeter wavelength ranges. This hints at an advanced stage of dust evolution. Pascucci et al. (2014) noted that they resolve circumstellar structure around CS Cha with $3.3 \mathrm{~cm}$ ATCA observations outside of 30 arcsec. Since it can be excluded that this emission stems from the disk itself, they conclude that it is likely a jet, which is launched from the disk at a position angle of $\sim 162^{\circ}$.

\footnotetext{
1 We note that in a recent study by Voirin et al. (2018), the distance to the Cha I cloud was estimated to be slightly larger at $179 \mathrm{pc}$. This is well covered by our uncertainties and we prefer to use the smaller distance for better comparability with previous studies until a direct distance measurement for CS Cha by Gaia becomes available.
}

We used the SPHERE (Spectro-Polarimetric High-contrast Exoplanet REsearch; Beuzit et al. 2008) extreme adaptive optics imager to study the circumstellar environment of CS Cha in polarized near-infrared light. Our goals were to resolve the disk cavity for the first time and to study potential features of dust evolution or planet disk interaction such as rings or gaps and spiral arms. In addition to our SPHERE observations we used archival high contrast data to strengthen our conclusions.

\section{Observations and data reduction}

\subsection{The initial SPHERE polarimetric observations}

CS Cha was first observed with SPHERE/IRDIS (Infra-Red Dual Imaging and Spectrograph; Dohlen et al. 2008) in differential polarization imaging mode (DPI; Langlois et al. 2014) in the $J$ band on February 17, 2017 as part of our ongoing program to understand dust evolution in transition disks via the distribution of small dust particles. Conditions during the night were excellent with clear sky and an average seeing in the optical of 0.6 arcsec and a coherence time of $\sim 5 \mathrm{~ms}$.

The (unresolved) central binary was placed behind a coronagraph with a diameter of 185 mas (Martinez et al. 2009; Carbillet et al. 2011). We used an integration time of $96 \mathrm{~s}$ in individual exposures and one exposure per half wave plate (HWP) position. A total of 11 polarimetric cycles were recorded with a combined integration time of $70.4 \mathrm{~min}$. In addition to the science data, we recorded star center frames at the beginning and end of the sequence as well as flux calibration frames and sky frames. For the star center frames a symmetrical waffle pattern was induced on the deformeable mirror that produces four satellites spots in the image. These spots can be used to accurately determine the position of the source behind the coronagraph (Langlois et al. 2013). For the flux frames the central source was offset from the coronagraph and a total of ten images were taken with an individual exposure time of $2 \mathrm{~s}$ and a neutral density filter in place to prevent saturation.

The data reduction generally follows the description given in Ginski et al. (2016); the main difference is the instrumental polarization and cross-talk correction. We give a short summary here. Our reduction approach uses the double difference method (Kuhn et al. 2001; Apai et al. 2004). For this purpose we first subtracted the ordinary and extraordinary beam to create individual $Q^{+}, Q_{-}, U^{+}$and $U^{-}$images, corresponding to HWP positions of $0^{\circ}, 45^{\circ}, 22.5^{\circ}$, and $67.5^{\circ}$. We then subtracted $Q^{-}$and $Q^{+}$(and $U^{-}$and $U^{+}$) to remove the instrumental polarization downstream from the HWP within SPHERE. This is carried out on a cycle by cycle basis before all resulting images are median combined to obtain the Stokes $Q$ and $U$ images. We also created a total intensity image, i.e., Stokes $I$, from our data. This is performed by adding in all cases ordinary and extraordinary beams and then median combining all resulting images over all polarimetric cycles. The Stokes $Q$ and $U$ images still contain residual instrumental polarization mainly induced by the VLT/UT3 mirror 3 and SPHERE mirror 4. To most accurately determine the angles and degree of linear polarization, it is necessary to correct for instrumental polarization and cross talk. For this purpose we used the detailed Mueller matrix model and correction method of van Holstein et al. in prep. (including telescope mirrors, instrument common path, and IRDIS itself). This model was calibrated using an unpolarized standard star and the SPHERE/IRDIS internal (polarized) calibration light source and was validated with polarimetric observations of the TW Hya disk. The correction was performed on each individual double difference image 


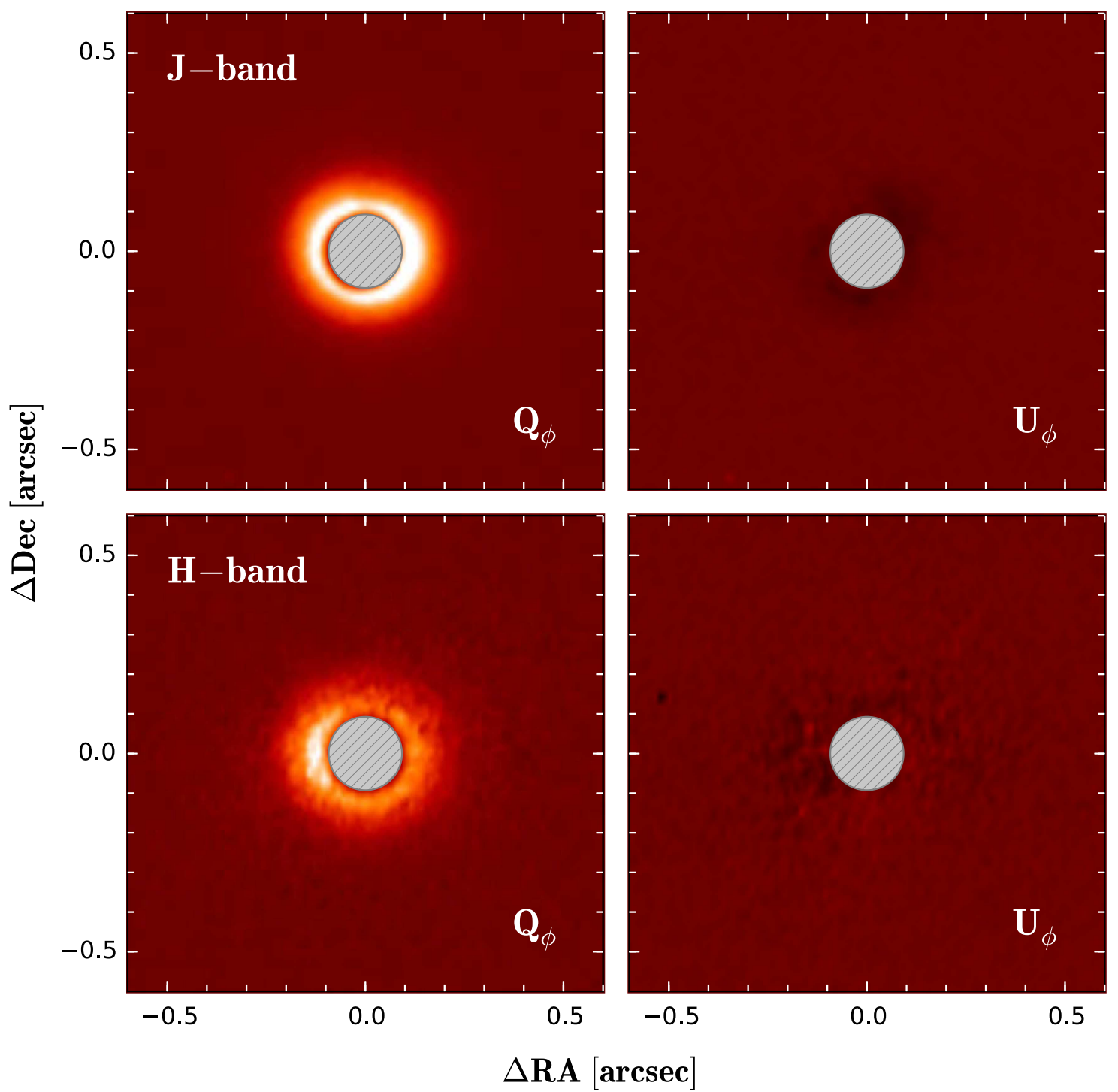

Fig. 1. 1 st row: reduced SPHERE DPI $J$-band $Q_{\phi}$ and $U_{\phi}$ as well as intensity image. North is up and east to the left. 2 nd row: same for our $H$-band observations. Color scale (linear) and stretch are the same for all $Q_{\phi}$ and $U_{\phi}$ images. We did not correct for the $1 / r^{2}$ drop-off in stellar irradiation. The gray hatched disk overplotted on the images shows the size of the coronagraph used.

taking the rotation angles of all optical components from the image headers into account. The instrument polarization model was successfully applied in several recent studies of circumstellar disks imaged with SPHERE, such as the cases of T Cha (Pohl et al. 2017), DZ Cha (Canovas et al. 2018), and TWA 7 (Olofsson et al. 2018), as well as for the observation of substellar companion polarization in the case of the HR 8799 system (van Holstein et al. 2017).

Finally we used the Stokes $Q$ and $U$ images to compute the radial Stokes parameters $Q_{\phi}$ and $U_{\phi}$ (see Schmid et al. 2006). The $Q_{\phi}$ image contains all azimuthally polarized flux as positive signal and radially polarized flux as negative signal. $U_{\phi}$ contains all flux with polarization angles $45^{\circ}$ offset from radial or azimuthal directions. In the case of single scattering by a central source, we expect all signal to be contained in $Q_{\phi}$ and thus $U_{\phi}$ can be used as a convenient noise estimate. This is typically a valid assumption for disks seen under low inclination (Canovas et al. 2015). We show our final reduced polarimetric images in Fig. 1. We show the total intensity image in Fig. 2.

In our polarimetric images we clearly detected a compact, low inclination circumstellar disk in scattered light around
CS Cha. Furthermore, we detected in our total intensity images a faint companion candidate approximately $1.3 \operatorname{arcsec}$ to the west of CS Cha. After inspection of the polarized intensity images at the companion position, it became apparent that we also detected the companion in polarized light. We show the final polarized intensity image including circumstellar disk and companion overlaid with the angle of linear polarization in Fig. 3. Since the companion was detected in polarized light and in total intensity we can calculate its degree of linear polarization. We discuss this in detail in Sect. 5.

\subsection{Archival NACO imaging data}

The CS Cha system was previously observed with VLT/NACO (Lenzen et al. 2003; Rousset et al. 2003) as part of a stellar and substellar multiplicity survey among young Chamaeleon members (see Vogt et al. 2012 for results of that survey). Observations were carried out on February 17, 2006, i.e., exactly 11 yr before our new SPHERE observations. The data was taken in standard jitter mode in the $K_{s}$ band. Integration time for each individual exposure was $1 \mathrm{~s}$, and 35 exposures were taken and co-added 

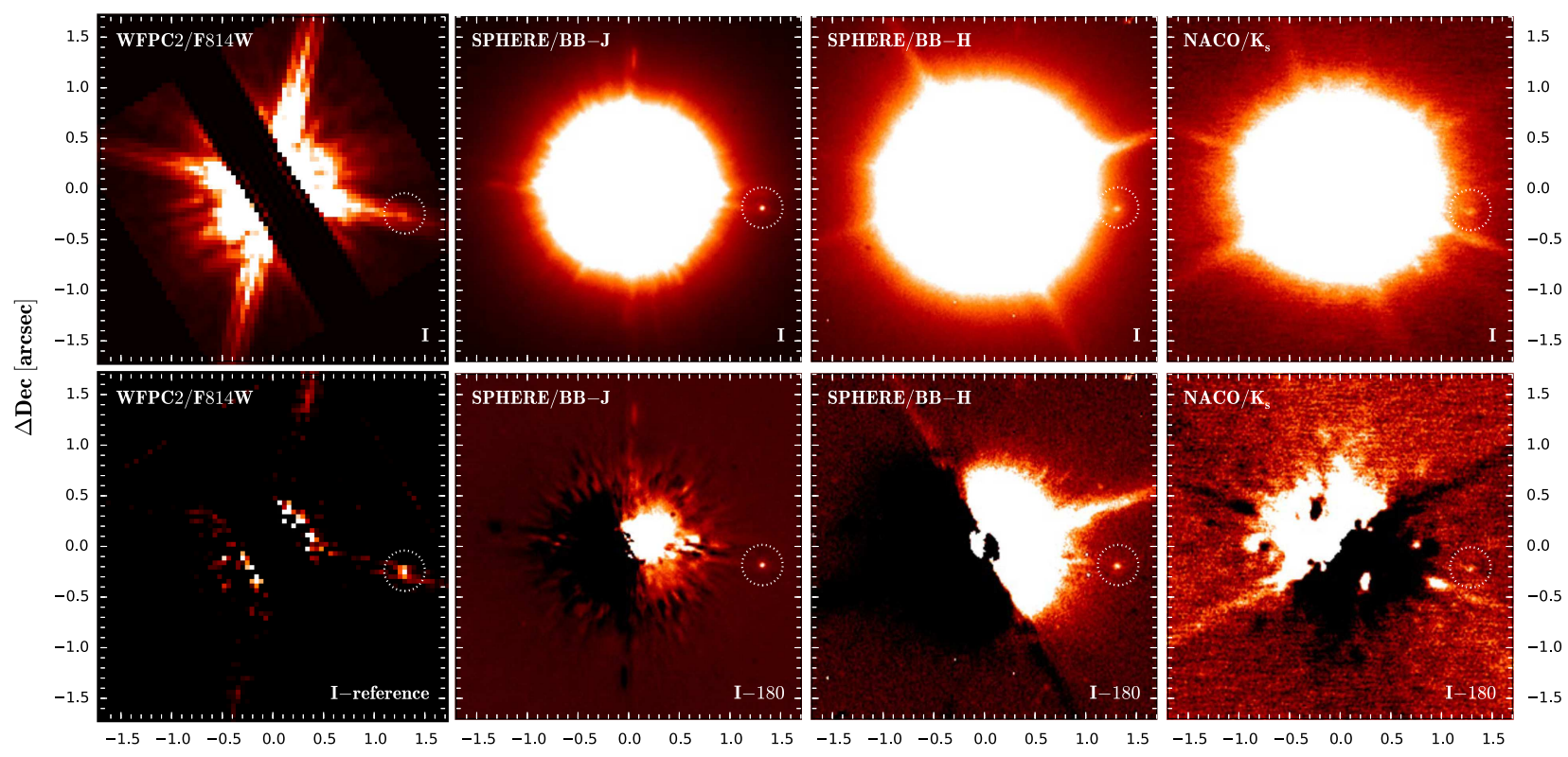

$\Delta \mathbf{R A}[\operatorname{arcsec}]$

Fig. 2. 1 st row: SPHERE $J$ and $H$-band intensity images as well as the NACO $K_{\mathrm{s}}$-band image and the WFPC2 F814W image of CS Cha. North is up and east to the left. In all images the position of the faint companion candidate is indicated by a white dashed circle. 2 nd row: same images as above but subtracted with a $180^{\circ}$ rotated version of themselves to remove the bright stellar halo. In the case of WFPC 2 we subtracted a reference star scaled to the flux of CS Cha to remove the bright stellar PSF and especially the bright diffraction spike on top of the companion position. In the WFPC2 images we removed the central columns containing the PSF peak since they were heavily saturated.

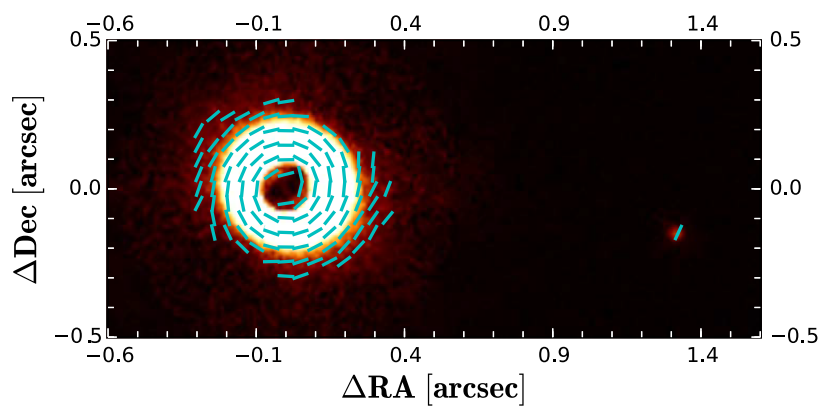

Fig. 3. Polarized intensity image of CS Cha and its companion in the $J$ band, after instrumental polarization correction. The circumbinary disk and the companion are well detected in polarized light. We overlayed the angle of the linear polarized light with light blue bars. The companion deviates by $\sim 20^{\circ}$ from an azimuthal polarization w.r.t. CS Cha indicating that it is intrinsically polarized and does not just scatter stellar light.

per jitter position. The total integration time of the data set was $11.7 \mathrm{~min}$.

We used ESO-Eclipse for the standard data reduction of the NACO data. This consisted of flat-fielding, bad pixel masking, and sky subtraction. The individual reduced images were then registered with respect to the central source and median combined.

In addition to standard data reduction, we removed the radial symmetric part of the stellar point spread function (PSF) by subtracting a $180^{\circ}$ rotated version of the image from itself. This was carried out to highlight faint companions at close angular separations and enable an accurate photometric and astrometric measurement without influence of residual stellar flux. The final reduced images are shown in Fig. 2. We redetected the faint companion candidate first seen in our SPHERE observations in the final reduced NACO image.

\subsection{Archival HST/WFPC2 observations}

In addition to the NACO archival observations, CS Cha was also observed with the Hubble Space Telescope Wide Field and Planetary Camera 2 (HST/WFPC2; Trauger \& WFPC2 Science Team 1994) on February 18, 1998. CS Cha was centered in the Planetary Camera subaperture of WFPC2 with an effective pixel scale of $46 \mathrm{mas} / \mathrm{pixel}$. The observations consisted of two exposures each in the F606W and the F814W filters, i.e., the WFPC2 equivalents of $R$ and $I$ band. Exposure times for the F606W filter were $8 \mathrm{~s}$ for the first exposure and $100 \mathrm{~s}$ for the second exposure with gain settings of 14 and 7, respectively. In the F814W filter the exposure times were $7 \mathrm{~s}$ and $80 \mathrm{~s}$ with the same gain setup. The innermost 2 pixels of the primary PSF and additional pixels along the central pixel readout column were saturated in all exposures. The data was reduced using the standard archival HST/WFPC2 pipeline.

To increase the detectability of faint point sources around the primary star, we subtracted a scaled reference star PSF from the long exposure images. As reference star we used the K5 star HD 17637, which was imaged for that purpose in the same program as the science data. As noted by Krist et al. (2000), the two main factors in achieving a good PSF subtraction result are a similar spectral type of the reference star and science target, and the placement of the reference star on the detector. Because of the under-sampling of the HST PSF, it is important to use a reference star that was imaged as close in detector position as possible to the science target. From the multiple images that were taken of HD 17637, we thus chose the image with the smallest angular separation from the position of the science target in both filters. Since the reference star and CS Cha both had a saturated PSF core, we could not use the PSF peak for scaling of the reference star PSF. We instead used an annulus along the unsaturated flanks of the PSF to compute the scaling factor.

After subtraction of the reference star, we detected a faint point source at the expected companion candidate position in 
the F814W images with a signal-to-noise ratio $(\mathrm{S} / \mathrm{N})$ of 5.0. The companion candidate was hidden under one of the bright diffraction spikes of the primary PSF. We show the subtracted and nonsubtracted image in Fig. 2. In the F606W data set we could not find a significant detection at the companion candidate position.

\subsection{NACO L-band follow-up observations}

To image CS Cha in the thermal infrared, we used VLT/NACO again. The observations were acquired on April 28, 2017 using the angular differential imaging (ADI) mode of NACO with the L' filter and the L27 camera following the strategy described by Chauvin et al. (2012). The NACO detector cube mode was also used for frame selection with exposure time of $0.2 \mathrm{~s}$. A classical dithering sequence was used with the repetition of five offset positions to properly remove the sky contribution. In the end, the typical observing sequence represented a total of 57 cubes of 100 frames each, i.e., a total integration time of $19 \mathrm{~min}$ for an observing sequence of $45 \mathrm{~min}$ on target. Two sequences of nonsaturated PSFs were acquired using a neutral density filter at the beginning and the end of each observing sequence to monitor the image quality. These data also served for the calibration of the relative photometric and astrometric measurements. The reduction of the ADI saturated dithered data cubes was performed with the dedicated pipeline developed at the Institut de Planétologie et d'Astrophysique de Grenoble (IPAG; Chauvin et al. 2012) providing various flavors of ADI algorithms. At the separation of the candidate, the background noise is the main source of limitation. Spatial filtering and simple derotation or classical ADI are therefore sufficient to process the ADI data. After final data reduction we did not detect the companion candidate in our NACO $L_{p}$-band data.

\subsection{SPHERE polarimetric follow-up observations}

Our initial SPHERE observations were followed up on June 18, 2017 with SPHERE/IRDIS DPI observations in the $H$ band with the goal to obtain $H$-band photometry of the companion candidate and to confirm its detection in polarized light. The conditions during the observations were overall poor. Even though the seeing in the optical was on average only 0.7 arcsec, the coherence time was very short on the order of $2 \mathrm{~ms}$ on average during the observations. This lead to a much poorer AO correction compared to the previous $J$-band observations.

The setup of the June observations was similar to the first set of observations in February. The central binary was again placed behind the 185 mas coronagraph. We used a slightly shorter individual exposure time of $64 \mathrm{~s}$ owing to the unstable weather conditions. We recorded a total of seven polarimetric cycles with a combined integration time of 29.9 min.

Data reduction was performed analogously to the previous $J$-band data set. We found that the circumstellar disk and the companion candidate were again detected in polarized light. We could also detect the companion in our stacked total intensity images. Final reduced images are shown in Figs. 1 and 2.

\section{Astrometric confirmation of the companion}

Since the companion was detected in our new SPHERE data and the archival NACO and HST data, we were able to to test whether the companion is comoving with CSCha. To ensure minimal contamination by the flux of the central star in the SPHERE and NACO images, we subtracted a $180^{\circ}$ rotated version of the images from the original (see Fig. 2). For the HST
F814W image we used the reference star subtracted image to determine the companion position.

In the case of the SPHERE and NACO images we used IDL starfinder (Diolaiti et al. 2000) to fit a reference PSF to the companion and extract its position in detector coordinates. As reference PSF we used the unsaturated stellar primary in the NACO image and the dedicated flux frames taken for the SPHERE images. Since the separation of the companion is smaller than the average isoplanatic angle at Paranal (see, e.g., Martin et al. 2000), no significant distortions of the companion PSF compared to the primary star PSF are expected. For the HST PSF under-sampling and residuals from the diffraction spike made PSF fitting problematic. Instead we used ESOMIDAS (Banse et al. 1983) to fit a two-dimensional Gaussian to the companion position. Measurements were repeated several times with various input parameters in terms of measuring box size and starting position to ensure that the fit converged well. We used the average value of all measurements as the final extracted companion position. We used the individual fitting uncertainties of the Gaussian fit as the uncertainty of the detector position of the companion. We ensured that this uncertainty was significantly larger than the standard deviation of multiple repeated measurements with various initial parameters.

To extract the stellar position, we used different approaches for the SPHERE, NACO, and HST data. For the NACO data no coronagraph was used, so we used the same approach to extract the stellar position as was used for the companion position. However, for SPHERE no direct measurement was possible since the central source is obscured by the coronagraph. Instead we used the center calibration frames to determine stellar position, as described in Langlois et al. (2013). Since we had multiple center frames taken at the beginning and end of the sequence, we used the deviation between the recovered positions as the uncertainty of the central source position measurement. For the HST image the primary star was heavily saturated with significant column bleeding, making a fit to the remaining stellar PSF difficult. Instead we fit linear functions to the positions of the diffraction spikes and used their intersection as stellar center position.

To translate the recovered detector position for the central binary and the companion into on-sky separation and position angle, our observations required an astrometric calibration. For the archival NACO data several binary stars were imaged as astrometric calibrators during the same night as the science data as part of the original program. The results of these astrometric calibrations, including the potential orbital motion of the binary calibrators, are given by Vogt et al. (2012).

For the SPHERE data, calibrators are regularly imaged during the ongoing SPHERE GTO survey. Primary calibrators are stellar clusters such as 47 Tuc, $\Theta$ Ori B, and NGC 6380. The results of these astrometric calibrations are given in Maire et al. (2016). In addition, detailed solutions for the geometric distortions were calculated by these authors. The instrument has proven to be extremely stable within their given uncertainties. We thus use their results for the broadband $\mathbf{J}$ filter to calibrate our data. We also use their distortion solution to correct geometric distortions in our SPHERE image. For the true north of the $J$ band data we use the more recent measurement published in Chauvin et al. (2017), done within a few days of our observations. The uncertainties for the SPHERE data include those of the detector coordinates of the central source and companion as well as the calibration uncertainty and the uncertainty of the distortion solution. Lastly, for the HST data we used the astrometric calibration provided in the image header. We list final results in Table 1. 
Table 1. Astrometric measurements and calibrations of all observation epochs.

\begin{tabular}{llcccc}
\hline \hline Epoch & Instrument & Pixel scale (mas/pixel) & True north $(\mathrm{deg})$ & Separation $(\operatorname{arcsec})$ & Position angle (deg) \\
\hline 1998.1339 & WFPC2 & $45.52 \pm 0.01$ & $31.69 \pm 0.005$ & $1.314 \pm 0.039$ & $258.26 \pm 1.21$ \\
2006.1311 & NACO & $13.24 \pm 0.18$ & $0.18 \pm 1.24$ & $1.299 \pm 0.018$ & $260.30 \pm 1.24$ \\
2017.1311 & SPHERE/IRDIS & $12.263 \pm 0.009$ & $-1.71 \pm 0.06$ & $1.314 \pm 0.002$ & $261.41 \pm 0.12$ \\
2017.4617 & SPHERE/IRDIS & $12.251 \pm 0.009$ & $-1.75 \pm 0.11$ & $1.319 \pm 0.001$ & $261.40 \pm 0.23$ \\
\hline
\end{tabular}
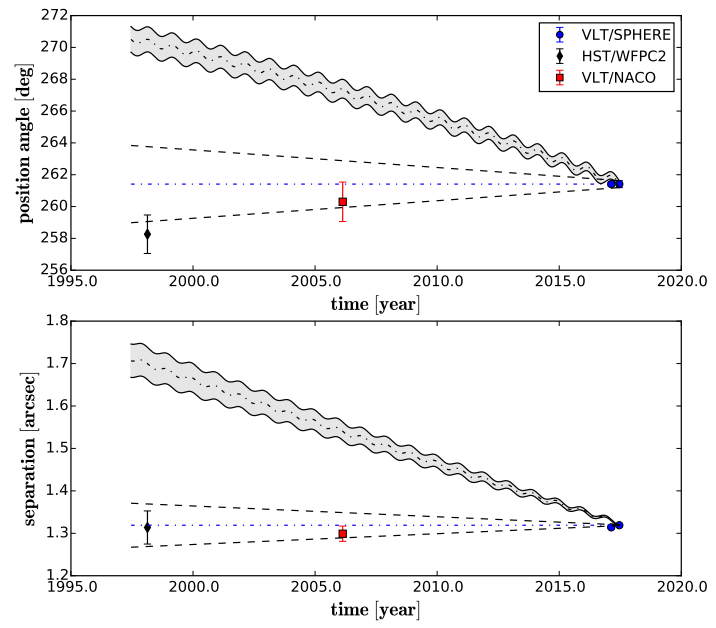

(a) PM from NOMAD catalog
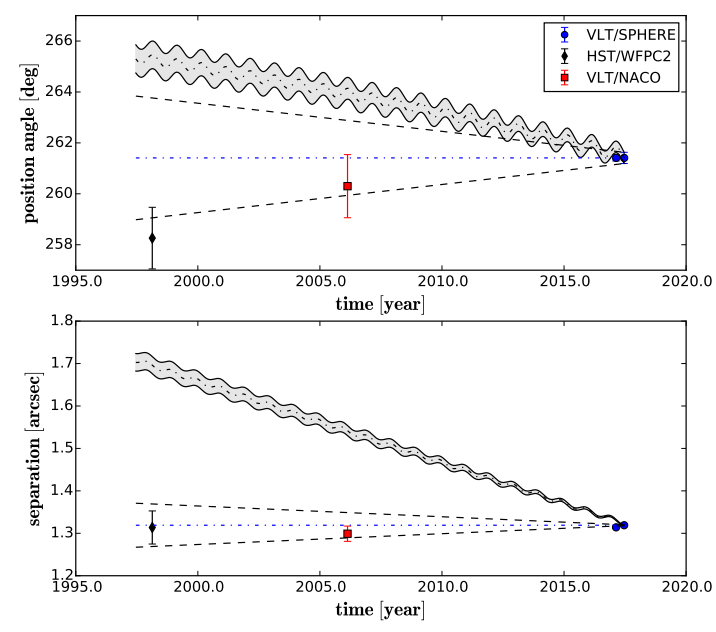

(b) PM from SPM4 catalog
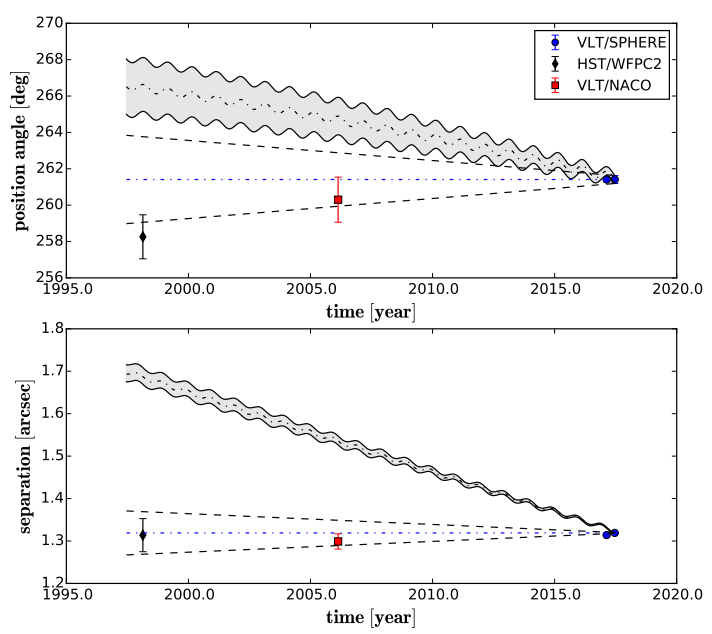

(c) PM from Smart et al. 2014

Fig. 4. Proper motion diagrams of the companion relative to CS Cha. The wobbled gray lines are the area in which a nonmoving background object would be expected. The "wobbles" are due to the parallactic displacement of such an object visible during the Earths revolution around the Sun. The dashed lines indicate the area in which a comoving companion would be located. The dashed lines take potential orbital motion into account assuming an inclination (circular face-on for the position angle and circular edge-on for the separation) and total system mass (1 $M_{\odot}$, i.e., this assumes that the mass of the companion is small compared to CS Cha). In all three diagrams the companion is comoving with CS Cha and thus in all likelihood gravitationally bound. We note that we see a small differential motion in position angle across our 19 yr observation baseline, which is consistent with a circular face-on (or close to face-on) orbit.

After we extracted the astrometry in all epochs, we measured the proper motion of the companion relative to CS Cha. The final results are shown in Fig. 4. We show three different diagrams, since the proper motion of CSCha is given with slightly different values in the NOMAD (Zacharias et al. 2004) and SPM4 (Girard et al. 2011) catalogs, and by Smart \& Nicastro (2014). In all three cases we can clearly reject the background hypothesis with $7.1-8.7 \sigma$ in separation and with $4.4-8.5 \sigma$ in position angle. Within the given uncertainties we observe no significant relative motion in separation over our $\sim 19$ yr baseline. However we observe relative motion in position angle, which is consistent with a circular face-on or low inclination orbit, i.e., with a similar inclination as is observed for the resolved circumbinary disk. Within the given error bars 
the companion is thus comoving with the primary stars. This is a very strong indication that the companion is gravitationally bound to CS Cha. In particular it is extremely unlikely that the companion is a blended extragalactic source, since such a source would have to move at very high velocity and would need to be by-chance aligned in proper motion and close to CS Cha. The probability for a blended Galactic source might be slightly higher. To quantify this we used the TRILEGAL (Girardi et al. 2005, 2012) population synthesis model to compute the number of expected Galactic sources in close vicinity of CS Cha. As input we gave the Galactic coordinates of CS Cha and the $J$-band magnitude of the companion as limiting magnitude. Following Lillo-Box et al. (2012) the number of expected objects can then be translated into a probability of finding a background object at a certain separation. Using this approach we find that the chance of a faint blended Galactic source within 1.3 arcsec of CS Cha is $0.4 \%$, i.e., improbable at the $2.9 \sigma$ level. Such a source would then still need to be by-chance aligned in proper motion with CS Cha making this scenario even less likely. One last concern might be that the companion could be a blended local source within the Cha I cloud but several pc behind CS Cha. For example Cambresy et al. (1998) found a number of very faint and highly embedded young stellar objects (YSOs) in Cha I. To test the likelihood of a by-chance aligned local source in Cha I we checked the dispersion of proper motions of known members. As input we used the catalog by Teixeira et al. (2000), which contains 29 such members, including CS Cha. We find that the dispersion in proper motion is quite high with $\sim 18 \mathrm{mas} / \mathrm{yr}$ in right ascension and $\sim 73 \mathrm{mas} / \mathrm{yr}$ in declination. In contrast we find that the companion shows no significant deviation from the proper motion of CS Cha in right ascension and only $3.6 \pm 1.8 \mathrm{mas} / \mathrm{yr}$ in declination, which can be well explained by orbital motion as mentioned earlier. In Fig. B.1, we furthermore show that the recovered colors of the companion do not match the YSO colors in Cha I by Cambresy et al. (1998). We can thus firmly exclude a blended local object as well. We overall conclude that the companion is in all likelihood gravitationally bound to CS Cha. We explore the orbital motion of the companion in detail in Sect. 7.1.

\section{Photometric measurements and detection limits}

\subsection{SPHERE and NACO photometry}

To understand the nature of the faint companion, we performed photometric measurements in all bands in which the companion was detected and derived upper limits for the nondetections. In our SPHERE $J$ and $H$-band epochs, and in the NACO $K_{s}$-band epoch, the companion was well detected but was still located close enough to CS Cha such that the background at the companion position is dominated by the bright stellar halo. We did not image PSF reference stars (nor was a PSF reference available for the archival NACO data), thus we assumed that the low frequency structure of the stellar halo is approximately radial symmetric. To remove this radial symmetric halo, we subtracted $180^{\circ}$ rotated versions of the images from themselves. The results are shown in Fig. 2. While strong signal remains within $\sim 0.5$ arcsec of CS Cha, the companion position appears free of strong residuals.

After this initial background subtraction we used IDL starfinder to perform PSF fitting photometry to measure relative brightness between the companion and CS Cha (the latter in the unsubtracted images). We used the flux calibration frames for the SPHERE observations to obtain an unsaturated reference PSF. For the NACO $K_{s}$-band image we used CS Cha itself as reference PSF since it was not saturated during the science sequence. Once a PSF fitting result was obtained we subtracted the companion from the data to check for strong residuals at the companion position. The results are given in Table 2 as differential magnitudes. To convert the differential $J, H$, and $K_{s}$ magnitudes to apparent magnitudes of the companion we used the corresponding 2MASS (Cutri et al. 2003) magnitudes of CS Cha as calibration. We then also list absolute magnitudes for which we assumed a distance of $165 \pm 30 \mathrm{pc}$. For conversion to physical fluxes we used a HST/STIS Vega spectrum and the filter curves of SPHERE and NACO.

We note that we find a clear systematic uncertainty in the SPHERE $H$-band observations induced by the poor observing conditions. In particular the coherence time of the atmosphere degraded during the sequence with longer values at the start than at the end of the sequence. However, our flux calibration frames were only taken at the end of the sequence, i.e., in the worst observing conditions, and thus have lower Strehl than the previous science images in the sequence. Thus using these images for the flux measurement of the companion during the whole sequence overpredicts the companion flux. To estimate this systematic effect we subdivided the science sequence into four equally long bins, which we reduced individually to detect the companion in each bin. We then measured the relative loss of signal in the companion due to changing weather conditions between all bins. We found a deviation of $0.46 \mathrm{mag}$ between the first and last bin. We consider this as an additional error term for the lower limit (since we know the direction of the effect) of the companion flux in the $H$ band.

As mentioned earlier the companion was not detected in our NACO $L$-band observation. We thus evaluated the detection limit of our observation. The detection performances reached by our observation were estimated by computing $2 \mathrm{D}$ detection limit maps at $5 \sigma$ in terms of $L_{p}$ contrast with respect to the primary. We computed the pixel-to-pixel noise within a sliding box of $1.5 \times 1.5 \mathrm{FWHM}$. The detection limits were then derived by taking the ADI flux loss using fake planet injection and the transmission of the neutral-density filter into account and were normalized by the unsaturated PSF flux. Our final detection limits map is shown in Fig. 5 and the computed detection limit at the companion position is given in Table 2. We used the WISE (Cutri et al. 2012) W1 magnitude as close proxy for the $L$-band magnitude of the primary star to convert contrast limits to apparent and absolute magnitude limits.

\subsection{WFPC2 photometry and detection limits}

To estimate the brightness of the companion in the WFPC2 F814W filter, several analysis steps were necessary. The primary star was saturated in the long exposure in which we detected the companion. To enable a relative measurement of the companion brightness we thus first determined the brightness of the primary star in the exposure. For this purpose we used TinyTim (Krist et al. 2011), which is a program designed to generate HST PSFs based on the instrument setup, target spectral type, time of observations, and position on the detector. We created a matching PSF for the WFPC2 F814W observations and then fitted this theoretical PSF to the unsaturated flanks of the CS Cha PSF by application of a scaling factor. We then used this scaled theoretical PSF for the relative brightness measurement with the companion.

The photometry of the companion in the WFPC2 image is challenging since it is contaminated by the bright diffraction spike of the primary star. Even after subtraction of a reference star, residuals of this diffraction spike are still visible around the 
Table 2. Photometric measurements of the companion.

\begin{tabular}{llcccccc}
\hline \hline Instrument & Filter & $\lambda_{c}(\mu \mathrm{m})$ & $\Delta \lambda(\mu \mathrm{m})$ & $\Delta$ mag & App. magnitude & Abs. magnitude & $F_{\lambda}\left(\mathrm{W} \mathrm{m}^{-2} \mu \mathrm{m}^{-1}\right)$ \\
\hline HST/WFPC2 & F606W & 0.5997 & 0.1502 & $>8.9$ & $>20.4$ & $>14.3$ & $<2.03 \times 10^{-16}$ \\
HST/WFPC2 & F814W & 0.8012 & 0.1539 & $9.81 \pm 0.48$ & $19.71 \pm 0.48$ & $13.62 \pm 0.62$ & $(1.37 \pm 0.76) \times 10^{-16}$ \\
SPHERE & BB-J & 1.245 & 0.240 & $10.05 \pm 0.21$ & $19.16 \pm 0.21$ & $13.07 \pm 0.45$ & $(6.31 \pm 1.39) \times 10^{-17}$ \\
SPHERE & BB-H & 1.625 & 0.290 & $9.20_{-0.15}^{+0.61}$ & $17.65_{-0.16}^{+0.62}$ & $11.56_{-0.43}^{+0.74}$ & $\left(2.54_{-1.95}^{+0.41}\right) \times 10^{-16}$ \\
NACO & $K_{s}$ & 2.18 & 0.35 & $9.21 \pm 0.16$ & $17.40 \pm 0.16$ & $11.32 \pm 0.43$ & $(3.44 \pm 0.56) \times 10^{-17}$ \\
NACO & $L_{p}$ & 3.80 & 0.62 & $>8.2$ & $>16.4$ & $>10.3$ & $<1.35 \times 10^{-17}$ \\
\hline
\end{tabular}

Notes. The apparent magnitudes in $J, H$, and $K_{s}$ band were calculated using the closest 2MASS magnitude of CS Cha as calibration. The apparent magnitude in the HST F814W filter was computed using the theoretical Vega magnitude of CS Cha in this band given its SED. The absolute magnitudes were computed from the apparent magnitudes assuming a distance of $165 \pm 30 \mathrm{pc}$. We give the central wavelength and spectral width of all filters along with the measurements. Spectral flux densities were computed using the filter curves of the instruments and a Vega spectrum taken with HST/STIS.

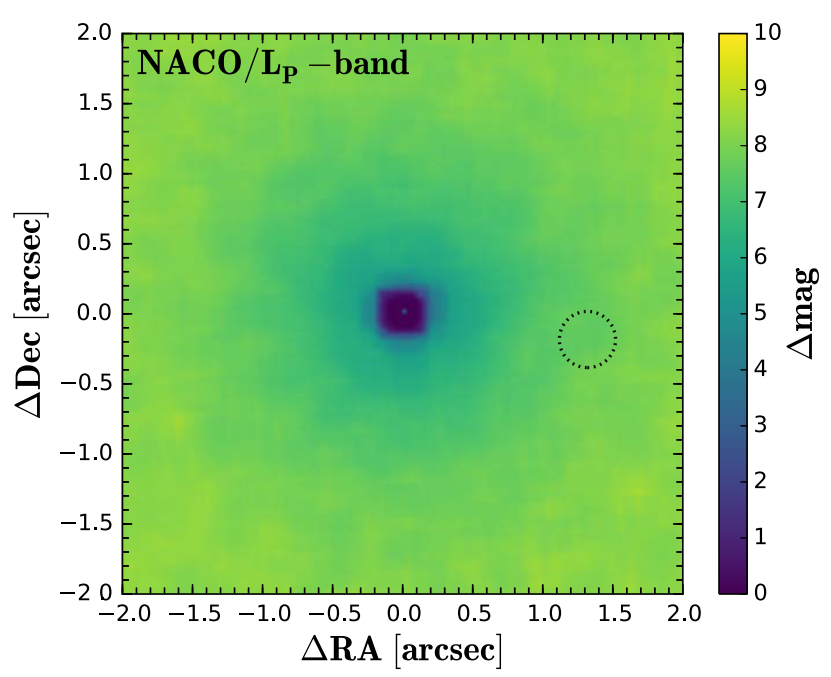

Fig. 5. Detection limit map derived from our NACO $L_{p}$-band observations. Detection limits are given in relative contrast to the primary star. We indicate the expected position of the companion with a black, dashed circle. The companion was not detected in these observations and should thus exhibit a contrast larger than 8.2 mag relative to CS Cha.

companion position. Because of the low $\mathrm{S} / \mathrm{N}$ of the detection and the under-sampling of the HST PSF, we decided against PSF fitting photometry in this case and instead applied aperture photometry. For this purpose we measured the flux of the companion in a $3 \times 3$ pixel box centered on the brightest pixel of the companion PSF. We then estimated the local background by measurements with the same box 3 pixels moved in radial direction toward and away from the central star along the diffraction spike. The average of both measurements was then subtracted from the companion measurement. To estimate the uncertainty of the background measurement we computed the standard deviation in the background apertures and multiplied it by the surface area of the aperture. In addition to the uncertainty of the background we took into account the read noise of the WFPC2 planetary camera for a gain setting of $7 \mathrm{e}^{-} / \mathrm{DN}$. We used a read noise of $5 \mathrm{e}^{-} /$pix. Overall the measurement is strongly dominated by the uncertainty of the background, which is a factor 4 higher than the estimated read noise. We give our result for the relative brightness measurement in magnitudes in Table 2.

To convert this relative measurement to an apparent magnitude of the companion, we determined the Vega magnitude of CS Cha in the F814W filter. For this purpose we calculated the

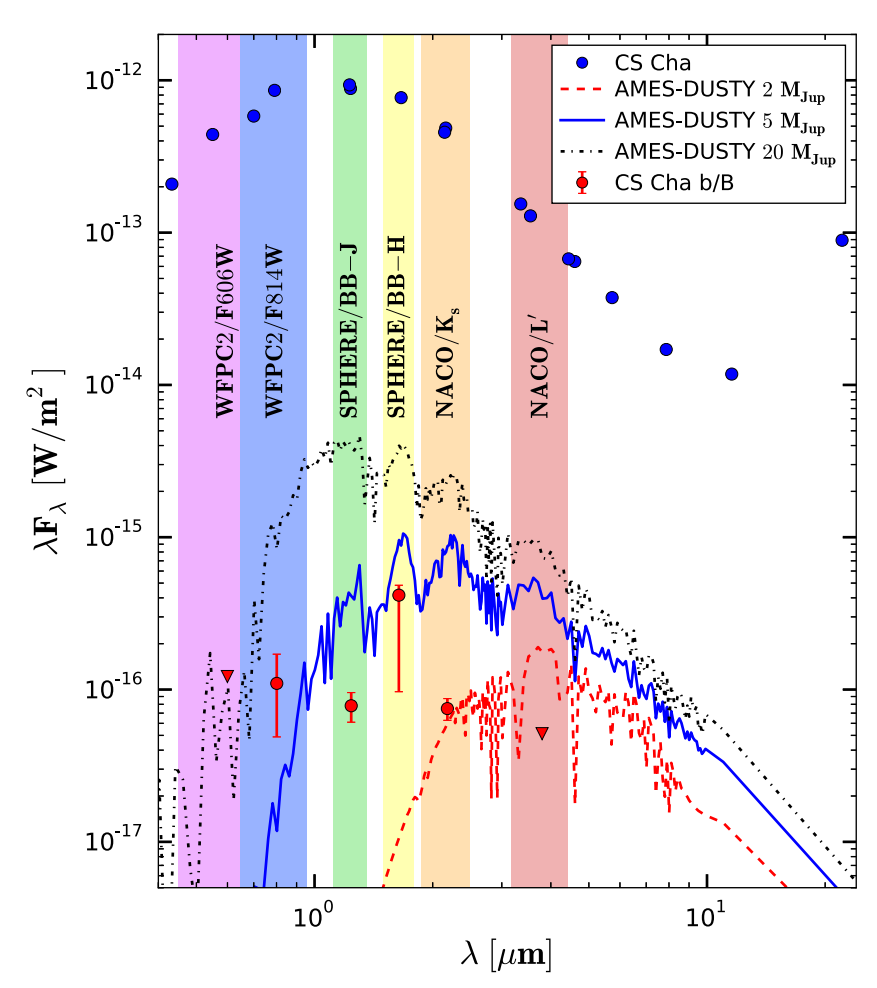

Fig. 6. Spectral energy distribution of CSCha (blue dots) and its companion (red dots and triangles). Downward pointing triangles denote upper limits. Spectral flux densities were computed from broadband photometry using a Vega spectrum and broadband filter curves. All values for the companion are given in Table 2.

total flux of CS Cha in the F814W filter using the filter curve and SED of CS Cha, shown in Fig. 6. We then converted this to a Vega magnitude by comparison with the flux of Vega in the same filter. We also give apparent and absolute magnitudes for the companion in Table 2, along with the physical flux of the companion in the F814W filter.

In the F606W filter the companion was not detected. We thus estimated detection limits at the companion position. For this purpose we measured the standard deviation at the companion position in a $3 \times 3$ pixel aperture. We again used TinyTim to create an unsaturated reference PSF scaled to the primary star brightness on the detector. Given the noise at the companion position and using the primary star as reference we then computed the limiting magnitudes for a $5 \sigma$ detection. The result is given in Table 2 . 


\section{Polarization of the companion}

Since we detected the companion both in the total intensity and polarized intensity images in our SPHERE/IRDIS $J$ - and $H$-band epochs, we were able to calculate the degree of linear polarization of the companion. For this purpose we used aperture photometry in both images in each band. We used aperture photometry over PSF fitting photometry due to the slight change in the companion PSF during the double difference steps of the DPI reduction. We checked in the $J$ band that PSF fitting photometry and aperture photometry give consistent results in the total intensity image. We found that the results were consistent within $0.01 \mathrm{mag}$. We used an aperture radius of 3 pix in the $J$ band, which corresponds to the full width at half maximum of 2.77 pix as measured by fitting a Moffat profile to the stellar PSF. In the $H$ band we used a value of 4 pix because of poorer observing conditions. As in the PSF fitting photometry, we first subtracted the radial symmetric bright stellar halo from the intensity images by rotating them $180^{\circ}$ and subtracting these images from themselves. We then estimated the local background with two subapertures in each band. In the $J$-band case, the companion is slightly contaminated by a stellar diffraction spike. We thus used two subapertures in radial direction along this spike. In the $H$ band we used two azimuthal subapertures at the same separation from the central star and offset by a few degrees from the companion position.

The measurement in polarized intensity was performed in the same way as in the intensity image. However, the measurements were actually performed in the Stokes $Q$ and $U$ images rather than in the combined polarized intensity image, since all signal becomes positive in this image and thus even background noise might give a spurious polarization signal.

We find a degree of linear polarization in the $J$ band of $13.7 \pm 0.4 \%$ and an angle of linear polarization of $153.0^{\circ} \pm 0.8^{\circ}$. Our $H$-band results are consistent with these measurements. We find a degree of linear polarization of $14.1 \pm 1.4 \%$ and an angle of linear polarization of $154.0^{\circ} \pm 2.9^{\circ}$. The uncertainties in the $H$-band measurements are higher due to the poorer $\mathrm{S} / \mathrm{N}$ compared to the $J$-band data. In both cases the error bars are strongly dominated by measurement uncertainties (due to photon, speckle and background noise), while the instrument model allows for a factor of $\sim 10$ higher accuracy.

We now need to investigate if the polarization of the companion is intrinsic to the object, i.e., either owing to scattered light from the primary stars or a central object within the companion itself, or if it is caused by interstellar dust between Earth and the CS Cha system. This is of particular importance since CS Cha is indeed located within close proximity or behind the Cha I dark cloud. Detailed optical polarization measurements of the region have been performed by Covino et al. (1997). In Fig. 7 we show their optical polarization map of the Cha I cloud region and superimpose the position of CS Cha. Using the nine stars in their study located closest to the position of CS Cha, we find an average maximum polarization degree of $6.7 \pm 1.7 \%$ at a peak wavelength of $0.65 \mu \mathrm{m}$. The average angle of polarization in the $I$ band that they measure for the same stars is $132.08^{\circ} \pm 12.9^{\circ}$. Using Serkowski's empirical law (Serkowski et al. 1975) we can extrapolate the expected polarization degree in the $J$ and $H$ band.

$p(\lambda)=p\left(\lambda_{\max }\right) \exp \left[-K \ln ^{2}\left(\lambda / \lambda_{\max }\right)\right]$.

Wherein $p(\lambda)$ is the polarization degree at the wavelength $\lambda$. The factor $K$ was empirically determined to be dependent on the peak wavelength of the polarization degree by Wilking et al. (1982).

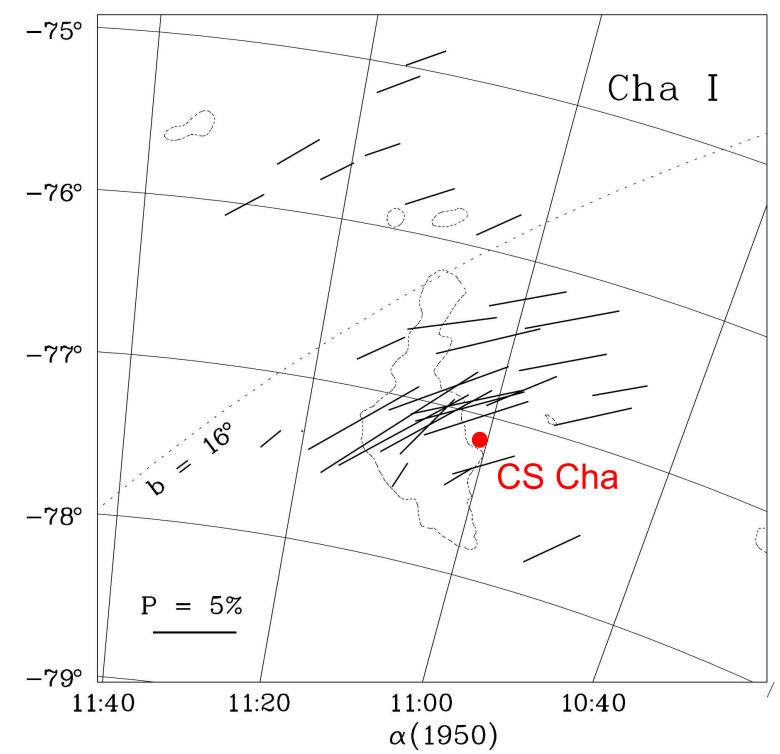

Fig. 7. Reproduction of Fig. 2 from Covino et al. (1997). Shown is a visual polarization map of the Cha I dark cloud using 33 stars they observed. Average angle and degree of polarization is indicated by the solid line vector field. We show the position of CS Cha in this map to indicate the expected degree of linear polarization introduced by the Cha I dark cloud in the optical.

$K=1.86 \lambda_{\text {max }}-0.1$.

Using these relations with the average values from Covino et al. (1997) we find that the degree of linear polarization could lie between $5.5 \%$ and $3.3 \%$ for the $J$ band and between $3.4 \%$ and $2.0 \%$ for the $H$ band. These degrees of polarization are significantly lower than what we find for the companion giving a first indication that the polarization of the companion is indeed intrinsic and not caused by interstellar dust.

To test this more rigorously we measured the degree of linear polarization of the unresolved primary stars. For this purpose we used an annulus at the bright speckle halo that marks the adaptive optics correction radius and contains only stellar light. We find that the primary stars have a degree of linear polarization of $0.57 \pm 0.28 \%$ in the $J$ band with an angle of polarization of $133.1^{\circ} \pm 8.2^{\circ}$. For the $H$ band we find similar values of $0.34 \pm 0.02 \%$ and $141.1^{\circ} \pm 5.4^{\circ}$ for the degree and angle of linear polarization, respectively. Both of these values are consistent with a previous measurement in the optical of CS Cha by Yudin (2000) who find a degree of linear polarization of $0.7 \%$ (but did not provide uncertainties). The degree of polarization that we find for the primary stars is much lower than suggested by the optical data by Covino et al. (1997) in combination with Serkowski's law. It could be that the average value we assumed is not a good proxy for the cloud density at the position of CS Cha or that CS Cha is located slightly in front of the cloud. In any case, the low degree of stellar polarization strongly suggests that the high degree of polarization found for the companion is intrinsic to the object and not caused by interstellar dust if we assume both objects are located at the same distance as suggest by their common proper motion.

This conclusion is additionally supported by the disagreement between the angle of polarization of the companion and that of stellar sources. In both bands the angle of linear polarization of the stellar binary is within $1 \sigma$ consistent with the average polarization angle in the region as determined by Covino et al. (1997). In our data we find that the companion 
polarization angle deviates by $\sim 22^{\circ}(2.6 \sigma)$ from the stellar polarization angle in the $J$ band and by $\sim 15^{\circ}(2.4 \sigma)$ in the $H$ band. Thus it seems again plausible that the cause for the polarization of the stellar binary and the companion are different and that the companion polarization is not caused by interstellar dust.

Given the angle of polarization of the companion, we can finally try to understand which is the dominating source of illumination, assuming polarization by single scattering of light. If the companion is primarily illuminated by the central stellar binary, we would expect its angle of linear polarization to be azimuthal with respect to the binary position. The expected angle of linear polarization for azimuthally scattered light at the companion position is $171.7^{\circ} \pm 0.1^{\circ}$. Comparing this to the more accurate angle of linear polarization in the $J$ band, we find a significant deviation of $18.7^{\circ} \pm 0.8^{\circ}$. We can thus conclude that the origin of the polarized light is not (entirely) single-scattered light emitted by the primary stars. It is of course still possible that the linear polarization that we measure is a superposition of scattered stellar and companion emission. However, given the angle of polarization we can already conclude that the companion object contains a central source massive enough that we can detect its emission.

Polarization can give us important information about the structure of the atmosphere of low-mass objects, as well as their direct environment. Polarization has indeed been measured for field brown dwarfs previously (see, e.g., Ménard et al. 2002; Zapatero Osorio et al. 2005; Miles-Páez et al. 2013), but was not detected so far for companions to nearby stars (see, e.g., Jensen-Clem et al. 2016; van Holstein et al. 2017). This is to the best of our knowledge the first time a faint and thus likely low-mass companion to a nearby star was detected in polarized light and its degree of polarization measured. We discuss the implications for the object in detail in Sect. 7.

\section{Circumbinary disk around CS Cha}

\subsection{Position angle and inclination}

As visible in Fig. 1, we resolve for the first time a small disk around the central stellar binary in the CS Cha system. The disk appears compact, smooth, and close to face-on. From our scattered light images we can extract the orientation of the disk. For this purpose we measured the disk diameter in radial disk profiles with orientations between $0^{\circ}$ and $360^{\circ}$ in steps of $2^{\circ}$. The resulting disk diameter versus disk orientation data was fitted with the corresponding value for an ellipse. The disk diameter was defined in our radial profiles as the separation between the two outermost points at which the disk flux reaches a certain threshold. To determine this threshold we measured the standard deviation of the background outside of the disk signal and set the threshold to a multiple of this standard deviation. In practice we found that there is a small dependency on the threshold value and the recovered disk orientation. We thus used multiples between 5 and 100 in steps of 2 and considered the recovered median values for disk inclination and position angle, and the standard deviation between these values, as the uncertainty of our measurement. Assuming a radial symmetric disk that only appears elliptical owing to its relative inclination toward us, we find a inclination of $24.2^{\circ} \pm 3.1^{\circ}$ and a position angle of $75.6^{\circ} \pm 2.2^{\circ}$ from our $J$-band observation. This disk position angle is well consistent with the position angle of the suspected jet emission of $\sim 162^{\circ}$ detected by Pascucci et al. (2014), since the jet position angle should be offset by $90^{\circ}$ from the disk major axis. The $H$-band observation has much lower $\mathrm{S} / \mathrm{N}$ than the $J$-band observation and suffers from convolution with a rather distorted PSF (see Fig. A.1). We find an inclination of $34.9^{\circ} \pm 10.6^{\circ}$ and a position angle of $86.1^{\circ} \pm 2.2^{\circ}$ for this data set. The $\sim 10^{\circ}$ larger position angle can be explained by the elongated PSF shape and orientation of this observation. We thus consider the $J$-band measurements as final values for inclination and position angle.

\subsection{Inner and outer radius}

To measure the outer radius of the disk we considered a radial profile along the major axis as determined in the previous section. We then computed the radial extent at which the disk signal is for the first time $5 \sigma$ above the image background value. We again used the $J$-band images because of their higher quality. We found an outer radius of scattered light of 337 mas, i.e., 55.6 au at a distance of $165 \mathrm{pc}$. This is consistent with the upper limit of $169 \mathrm{au}$ given by Dunham et al. (2016) from their unresolved ALMA observations. We note that we are only tracing small dust at the disk surface, therefore it is possible that the disk has a larger size but is partially self shadowed. Another possibility is that the disk outer extent is larger, but that it is below the noise floor in our images owing to the $1 / r^{2}$ drop-off of the stellar irradiation.

We show an azimuthally averaged radial profile of the disk in Fig. 8. In this profile a decline in brightness inside of $\sim 115$ mas is visible. To investigate whether this is a tentative detection of a cavity, we compared the radial disk profile with a model profile of the coronagraph attenuation. The NIR APLC coronagraph normalization profile was calculated based on IRDIS DB_H23 dual-band imaging observations of the $0.6^{\prime \prime}$ diameter disk of Ceres, performed on the December 14, 2016. This was carried out in the N_ALC_YJH_S coronagraph imaging mode and the Ceres disk was nodded off-center by 490 mas to provide a non-coronagraphic reference. This was used to produce a 2D attenuation profile of the coronagraph for an extended, incoherent source. Monochromatic Fourier modeling of the threeplane APLC coronagraph was also performed, using the APO1 SPHERE amplitude apodiser, ACL2 (185 mas diameter) focalplane mask, and NIR Lyot stop including dead actuator masks (Guerri et al. 2011; Sauvage et al. 2016). This model confirmed that the observed Ceres attenuation profile is nearly diffractionlimited and azimuthally symmetric. The radial profile outside of 85 mas is dominated by direct throughput of the target, while that inside 85 mas is dominated by internally scattered light in the instrument (for full results see Wilby et al. in prep.). The close agreement between the forward model and observed data allows the H23-band profile to be extrapolated to $J$ band via an equivalent model at $1.26 \mu \mathrm{m}$. This was then used to correct the radial CS Cha profile for coronagraph attenuation.

As visible in Fig. 8, after the correction with the coronagraph throughput profile, no significant decline in flux is visible outside the coronagraphic mask. We can thus put an upper limit on the size of the inner cavity of the CS Cha disk of $15.3 \mathrm{au}$ (92.5 mas at $165 \mathrm{pc}$ ) from the scattered light imaging (tracing small dust grains).

\section{The nature of the companion}

To understand the nature of this new companion, we compare its SED to known substellar objects in Chamaeleon and theoretical model atmospheres. We then use the astrometry over a $19 \mathrm{yr}$ baseline to determine if it is possible to constrain the companion mass from the orbital motion. Finally we use our own radiative transfer models to explain the photometry and degree of linear polarization of the companion. 


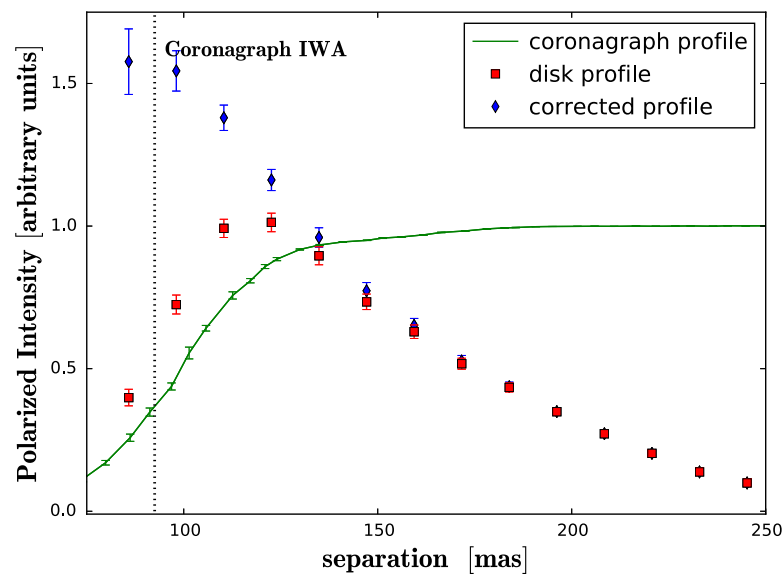

(a)

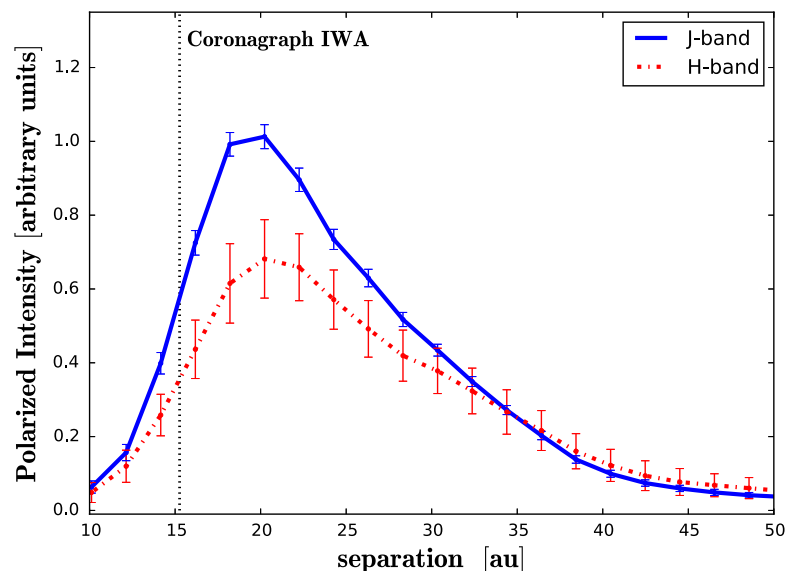

(b)

Fig. 8. Left: azimuthal average of the polarized intensity profile of the circumbinary disk around CS Cha in the $J$ band (red squares). The profile was measured in the $Q_{\phi}$ image, while the estimated uncertainties were determined in the $U_{\phi}$ image. We indicate the radius of the coronagraphic mask with the black dotted line. In addition, we show the throughput curve of the coronagraph used as discussed in Sect. 6.2 (green solid line). Finally we show the azimuthal disk profile corrected by the coronagraph throughput (blue diamonds). Right: azimuthal average of the polarized intensity profile of the circumbinary disk around CS Cha in the $J$ band (blue solid line) and the $H$ band (red dash-dotted line). Angular separations were converted to projected separations using the distance of $165 \mathrm{pc}$.

\subsection{A planetary mass object on a wide orbit?}

\subsubsection{Photometry}

In Fig. 6 we show the measured SED of the companion, and compared it with theoretical models of low-mass substellar objects calculated with the Phoenix (Helling et al. 2008) atmosphere code via the AMES-Dusty models (Chabrier et al. 2000; Allard et al. 2001) for an age of $2 \mathrm{Myr}$ as input. We tentatively explored a mass range between $2 M_{\text {Jup }}$ and $20 M_{\text {Jup }}$. The closest fit is achieved with a $5 M_{\text {Jup }}$ planet corresponding to an effective temperature of $1700 \mathrm{~K}$. However, it is clearly visible that even this best fit does not properly explain the measured photometry of the companion. While the J-H color may be explained by such an object (taking some redward shift due to extinction by circumplanetary material into account), the model clearly overpredicts the flux in the $K$ and $L$ bands by an order of magnitude. A significantly lower mass object of $2 M_{\text {Jup }}$ corresponding to an effective temperature of $1100 \mathrm{~K}$ could explain the $K$-band photometry, but still significantly overpredicts the $L$-band flux and is not compatible with the flux in the shorter wavelengths. Generally there is no model that can explain all photometric data points and upper limits. This is a strong indication that we are looking at an object that is either significantly more complex than a "naked" planetary photosphere, or that the object (or the primary) is for some reason strongly variable. Variability is indeed possible since all observation epochs have been taken months and sometimes decades apart.

One explanation for the peculiar shape of the SED could be a companion with a small (unresolved) surrounding disk. There are in fact two comparably faint objects in Chamaeleon known around which a circumplanetary disk is expected. One of these objects is the wide direct imaging companion to the young T Tau star CT Cha (Schmidt et al. 2008). CT Cha b shows $\mathrm{Pa} \beta$ emission in the $J$ band (Schmidt et al. 2008) and strong $\mathrm{H} \alpha$ emission in the $R$ band (Wu et al. 2015); both of these are strong indicators for ongoing accretion of material on the companion. The companion mass is estimated to be 9-35 $M_{\text {Jup }}$ with a temperature range between $2500 \mathrm{~K}$ and $2700 \mathrm{~K}$ (Schmidt et al. 2008; Bonnefoy et al. 2014; Wu et al. 2015). We show

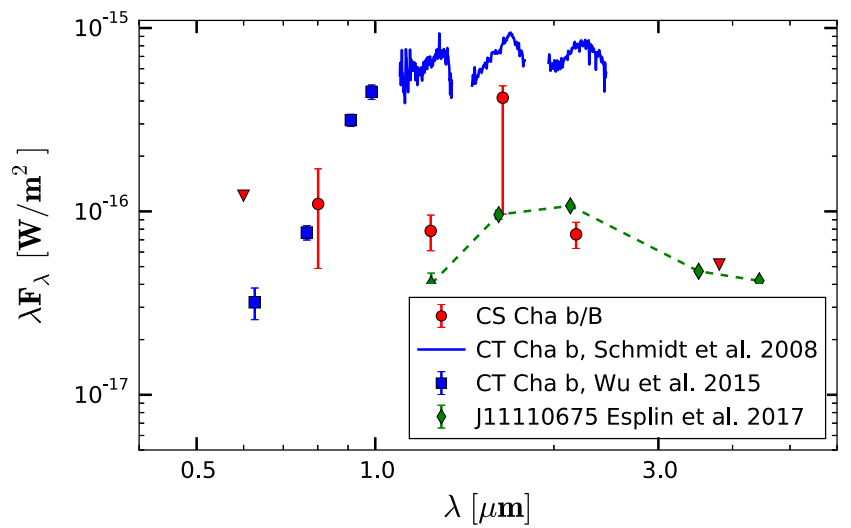

Fig. 9. Spectral energy distribution of the CSCha companion (red dots and triangles). Downward pointing triangles denote upper limits. We show the known substellar companion CTChab (Schmidt et al. 2008; Wu et al. 2015) and the free floating planetary mass object in Chamaeleon Cha J11110675-7636030 (Esplin et al. 2017) for comparison.

the near-infrared spectrum of CT Cha b along with optical photometry in Fig. 9. We overplot the photometry of the companion to CS Cha for comparison. While $R$-, $I$-, and $H$-band photometry are comparable in both objects, the $J$ - and $K$-band fluxes of CT Cha b are significantly larger than for the CS Cha companion. A second comparison object is the recently discovered free floating planetary mass object Cha J11110675-7636030 (Esplin et al. 2017), for which we also show available photometry in Fig. 9. Assuming an age range of 1-3 Myr and using a variety of planet evolutionary models, Esplin et al. (2017) have found a mass range of 3-6 $M_{\text {Jup }}$ for this object. They note that the mid-IR photometry suggests the existence of excess emission best explained by circumplanetary material. The object shows $J-K$ colors similar to the CS Cha companion and is also consistent with the $L$-band nondetection. The $H$-band photometry of both objects, on the other hand, differs significantly. For both comparison objects CT Cha b and Cha J11110675-7636030 we have no information on the geometry of the surrounding circumplanetary 


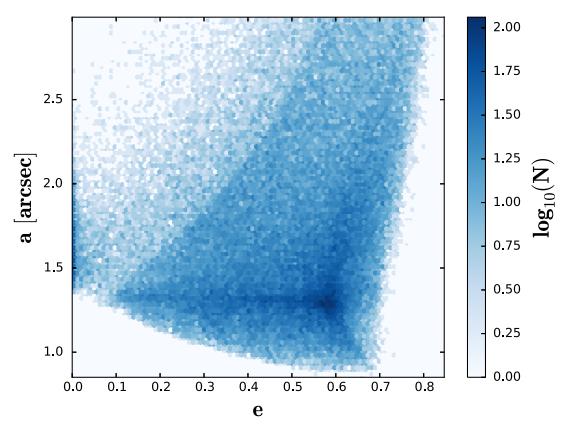

(a)

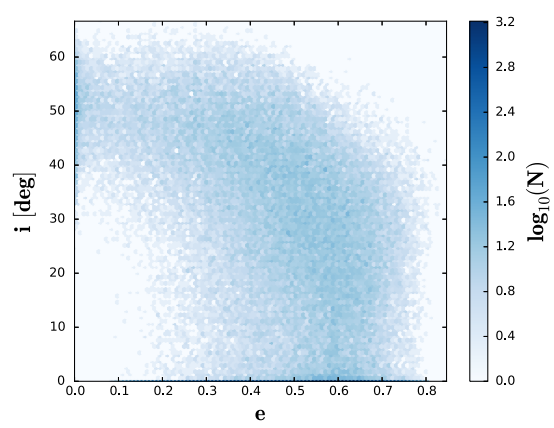

(b)

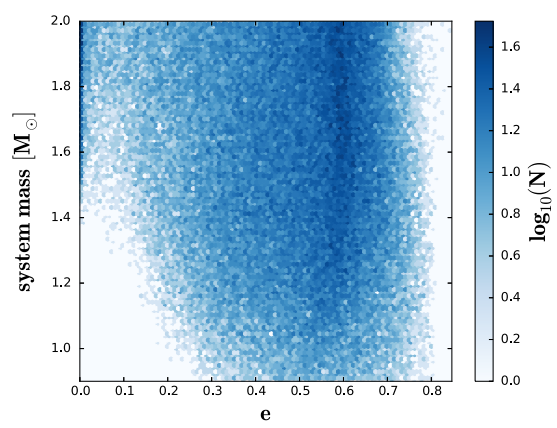

(c)

Fig. 10. Left: semimajor axis vs. eccentricity distribution of all recovered orbit solutions for the companion following the LSMC approach. Shown are the $1 \%$ best fitting orbits. Middle: same as left, but for eccentricity vs. inclination of the orbital plane. Right: same as left, but for eccentricity versus total system mass.

material. In particular we do not know the inclination of these inferred disks. It is possible that the companion around CS Cha is indeed more massive than both these objects, but is strongly extincted by a very inclined circum-companion disk. This scenario is indeed also supported by the high degree of linear polarization that we find for the companion. We thus explore several models with circum-companion material in Sect. 7.2.

\subsubsection{Astrometry}

Since we have an observational baseline of $\sim 19 \mathrm{yr}$, we attempted to fit the orbital motion of the companion around the primary stars. For this purpose we used the least-squares Monte Carlo (LSMC) approach as described in Ginski et al. (2013). We generated $10^{7}$ random orbit solutions from uniform priors and then used these as starting points for a least-squares minimization with the Levenberg-Marquardt algorithm. In contrast to Ginski et al. (2013), we did not assume a system mass but left it as a free parameter. To limit the large parameter space we constrained the semimajor axis to values between $0.5 \operatorname{arcsec}$ and 3.0 arcsec. This seems justified given the current position of the companion at $\sim 1.3$ arcsec and the fact that we see no significant change in separation between astrometric epochs. In addition, we limited the total system mass to values between $0.9 M_{\odot}$ and $2.0 M_{\odot}$. The lower end of this mass interval is determined by the lower limit of the combined mass of the central binary star, i.e., in this case the companion mass would be small compared to the primary mass in the planet or brown dwarf regime. The upper end is given by twice the upper limit of the central binary mass, i.e., in this case the companion would have roughly one solar mass. We do not expect the companion to be more massive than the primary stars, since the resolved circumbinary disk would otherwise likely be truncated to an even smaller outer radius.

In Fig. 10 we show the resulting semimajor axis, inclination, and mass versus eccentricity distributions of the $1 \%$ best fitting orbits. Since the uncertainties of the NACO and HST epochs are large compared to the SPHERE measurements, the fits are strongly dominated by the latter.

We find that the current astrometric epochs do not allow for constraint of the mass of the companion, since we find valid orbital solutions for the full range of input masses. However, we can make a few observations about the system architecture. If the companion is indeed a Jovian planet or brown dwarf, then we can conclude that it must be on an eccentric orbit with the lower limit of the eccentricity between 0.2 and 0.26 depending on the masses of the central stars. In fact the total system mass should be above $1.4 M_{\odot}$ to allow for circular orbits. In this case the companion would be a low-mass star with a mass between $0.4 M_{\odot}$ and $0.5 M_{\odot}$. Independent of the mass, we find an upper limit for the eccentricity of 0.8 . This upper limit is, however, introduced by our artificial cutoff of the semimajor axis at 3 arcsec. If we allow for larger semimajor axes, then we find even more eccentric orbits. This correlation between semimajor axis and eccentricity is indeed common for orbits that are not well covered with observations (e.g., Ginski et al. 2014). Overall we find a peak of the eccentricity at $\sim 0.6$. The vast majority of these eccentric orbits exhibits a face-on inclination.

It is interesting to investigate if coplanar orbits with the resolved circumbinary disk are possible since this could give an indication of the formation history. We find that such coplanar orbits indeed exist. However, regardless of the total system mass there are no circular $(e=0)$ coplanar orbits recovered. Overall the distribution of the total mass and eccentricity closely match the non-coplanar case.

In Fig. 11 we show the three best fitting orbit solutions that were recovered by our LSMC fit as well as the best fitting solutions for a circular, coplanar, and low-mass (companion mass below $0.03 M_{\odot}$ ) orbit. The respective orbital elements are given in Table 3. The best fitting orbits are not coplanar and exhibit eccentricities between 0.41 and 0.63 . Since most of these orbits are seen face-on there would be a significant misalignment between the inclination of the resolved circumbinary disk and the orbital plane and a misalignment with a putative highly inclined circum-companion disk. Such spin-orbit and spin-spin misalignments in multiple systems are indeed predicted by hydrodynamic simulations of stellar formation in clusters (see, e.g., Bate 2012,2018 ) and were more recently observed in multiple systems with ALMA (see the case of IRAS 43, Brinch et al. 2016). The total system masses for the best fitting orbits lie between $1.28 M_{\odot}$ and $1.84 M_{\odot}$, which puts the companion in the low stellar mass regime. However, we stress that lower (e.g., planetary) masses for the companion cannot be ruled out with the existing astrometry. One example for an orbital solution that fits the astrometry and requires only a companion mass below $0.03 M_{\odot}$ is shown in Fig. 11. In general these best fitting orbits may still change significantly with the availability of new high precision astrometric epochs in the future. Thus while the recovered distributions of orbital elements are meaningful, we caution against overinterpreting these specific orbit solutions. 


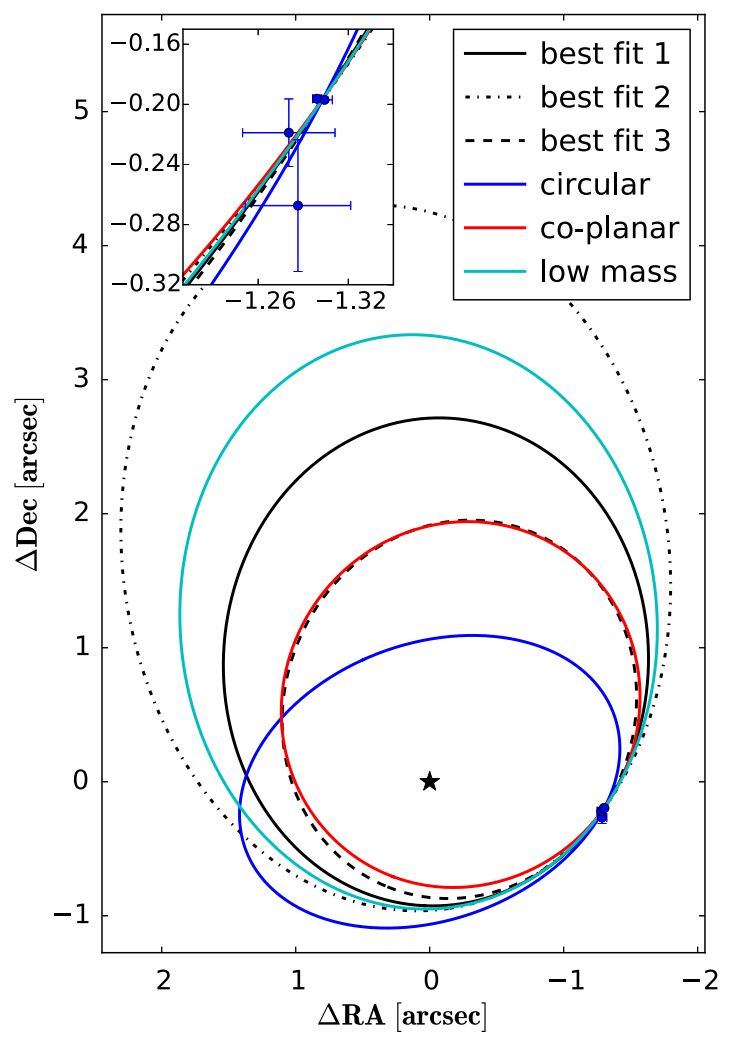

Fig. 11. Best fitting orbits to the current astrometry of the companion as recovered by our LSMC fit. The inset in the upper left is zoomed-in on the data points.

Table 3. Orbit elements of the three best fitting orbits shown in Fig. 11, as well as the best fitting circular (c.), coplanar (c.-p.), and low-mass (1.m.) orbits.

\begin{tabular}{lcccccc}
\hline \hline & 1 & 2 & 3 & c. & c.-p. & $1 . \mathrm{m}$. \\
\hline$a\left[{ }^{\prime \prime}\right]$ & 1.82 & 2.65 & 1.43 & 1.46 & 1.48 & 2.14 \\
$m\left[M_{\odot}\right]$ & 1.66 & 1.28 & 1.84 & 1.96 & 1.96 & 1.02 \\
$e$ & 0.49 & 0.64 & 0.41 & 0 & 0.45 & 0.56 \\
$P[\mathrm{yr}]$ & 4048.4 & 8076.2 & 2667.0 & 2678.6 & 2731.6 & 6579.6 \\
$i[\mathrm{deg}]$ & 0 & 0 & 0 & 45.2 & 21.6 & 0 \\
$\Omega[\mathrm{deg}]$ & 200.0 & 185.4 & 190.0 & 110.1 & 75.3 & 184.0 \\
$\omega[\mathrm{deg}]$ & 337.0 & 3.3 & 327.7 & 275.7 & 83.3 & 0 \\
$T_{0}[\mathrm{yr}]$ & 1651.9 & 1668.1 & 1602.1 & 3011.5 & 4363.7 & 1593.6 \\
\hline
\end{tabular}

\subsection{Detection of a circum-companion disk?}

To test whether the peculiar measurements of the companion can be explained by a substellar object surrounded by a disk, we aimed to model its photometric (SED) and polarimetric (degree of polarization) properties via the radiative transfer code RADMC3D. In all our models, we consider astronomical silicates (Weingartner \& Draine 2001) and use a single dust size for simplicity. We again consider the AMES-DUSTY atmosphere models as input for the central object. We run three different families of model.

(a) Our first model includes a disk around a substellar companion. The circumplanetary disk extends up to 2 au. This maximum outer radius was inferred from the fact that we do not resolve the companion. Its density is described as

$\rho_{\text {disk }}(r, z)=\frac{\Sigma(r)}{\sqrt{2 \pi} H_{\mathrm{p}}(r)} \exp \left(\frac{-z^{2}}{2 H_{\mathrm{p}}(r)^{2}}\right)$,
Table 4. Radiative transfer model parameters.

\begin{tabular}{lccc}
\hline \hline $\begin{array}{l}\text { Model } \\
\text { parameters }\end{array}$ & (a) Disk & (b) Envelope & $\begin{array}{c}\text { (c) Disk } \\
\text { and envelope }\end{array}$ \\
\hline$M_{\text {comp }}\left[M_{\text {Jup }}\right]$ & $5 / 20$ & 5 & 20 \\
$T_{\text {eff }}[\mathrm{K}]$ & $1580 / 2500$ & 1580 & 2500 \\
$R_{\text {comp }}\left[R_{\odot}\right]$ & $0.17 / 0.25$ & 0.17 & 0.25 \\
$R_{\text {in }}[\mathrm{au}]$ & 0.003 & 0.003 & 0.003 \\
$R_{\text {out }}[\mathrm{au}]$ & 2 & 2 & 2 \\
$M_{\text {disk }}\left[M_{\odot}\right]$ & $1.9 \times 10^{-7}$ & - & $1.9 \times 10^{-7}$ \\
$M_{\text {env }}\left[M_{\odot}\right]$ & - & $8.5 \times 10^{-9}$ & $2.3 \times 10^{-10}$ \\
$\rho_{0}\left[\mathrm{~g} / \mathrm{cm}^{3}\right]$ & - & $1 \times 10^{-16}$ & $5 \times 10^{-17}$ \\
$H_{\mathrm{p}}\left(R_{\text {out }}\right) / R_{\text {out }}$ & 0.18 & - & 0.18 \\
$\zeta$ & 0.25 & - & 0.25 \\
$p$ & -1 & - & -1 \\
$q$ & - & -1 & -1 \\
\hline
\end{tabular}

where $\Sigma(r)$ is the surface density and $H_{\mathrm{p}}(r)$ is the pressure scale height. Both quantities are described as power laws with radius, exponents $\zeta$ (flaring), and $p$. The model parameters are given in Table 4. The model has a complex parameter space, which we explored qualitatively by varying the mass and thus the luminosity of the central object, grain size, disk mass, and inclination. To produce a significant level of polarization (above $5 \%$ ), the disk must be strongly inclined (seen close to edge-on), in turn extinguishing the thermal emission from the companion and innermost disk radii. To match the SED at high inclinations we thus must increase the mass of the central object. An increase in disk mass has an effect that is similar to an increase of the inclination on the SED of the central object. Finally the grain size can be varied to modulate the polarization efficiency. We considered grain sizes of $0.5 \mu \mathrm{m}, 1 \mu \mathrm{m}$, and $2 \mu \mathrm{m}$. We show a sketch of the model along with the best fitting results for a $5 M_{\text {Jup }}$ and a $20 M_{\text {Jup }}$ companion in the left column of Fig. 12. For the lower mass we require a low disk inclination to get enough flux of the companion in the near-infrared. However, this model underpredicts the $I$-band flux, overpredicts the $L$-band flux, and does not reveal significant polarization. For the higher mass we find a much better fit. We can increase the disk inclination to much higher values, which match the $J$ - and $H$-band polarization well. Furthermore, the resulting SED is a close fit to all photometric measurements of the companion, excluding the $H$ band. We have also investigated whether we can derive an upper mass limit for the companion by placing a $72 M_{\text {Jup }}$ companion in the center of the disk. We found that even for high inclinations, such a model severely overpredicts the $K$ - and $L$-band flux.

We note that in this model we do not consider the binary as a source of irradiation. The only way that the companion would scatter a significant amount of light from the central binary at that distance would be if it stands outside of the plane of the circumbinary disk. Otherwise the light from the central binary would be blocked by the disk. We therefore tested the same model, but with an additional irradiation source (the central binary) at 214 au, and after placing the companion and disk outside of the plane of the circumbinary disk. We find that the scattered light signal from the central binary alone is between 2-3 orders of magnitude fainter than our measurements (see Fig. C.1 for comparison). It thus only has a marginal influence on our modeling results and was ignored for simplicity.

(b) We then changed our models to test a different geometry, and considered an envelope of dust grains surrounding the 

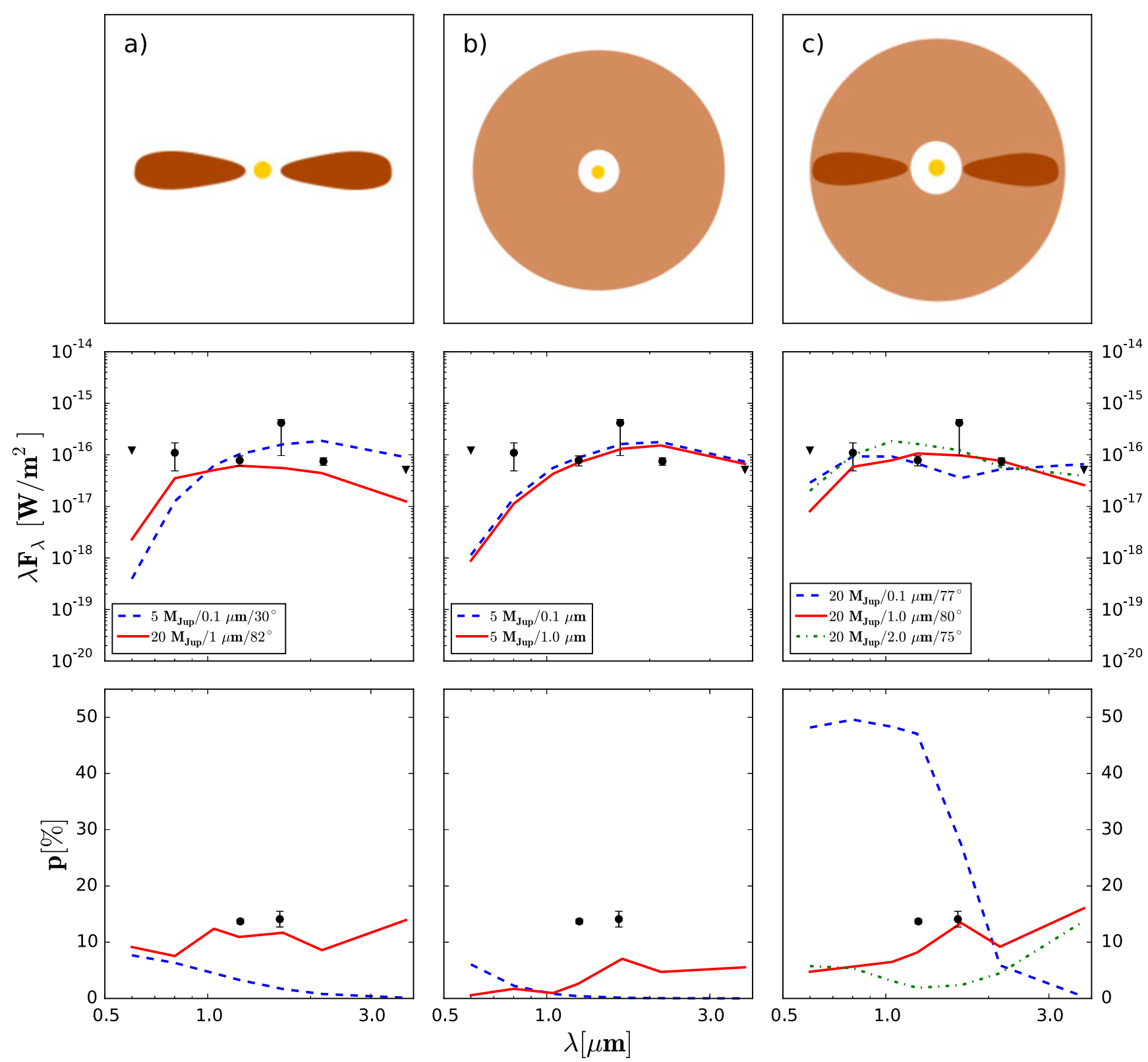

Fig. 12. 1st row: sketches of the model families described in Sect. 7.2. We show in all cases a cross section. 2nd row: photometry of the companion along with the model photometry for model parameters. The legend gives information about the assumed companion mass, size of the considered dust grains, and circumplanetary disk inclination (in models $a$ and $c$ ). $3 r d$ row: same as the second row, but for degree of linear polarization. Colors and line styles represent the same models as in the previous row.

companion, as this should enhance the amount of scattered light. The density structure is given by

$\rho_{\mathrm{env}}(r, z)=\rho_{0}\left(\frac{\sqrt{r^{2}+z^{2}}}{\sqrt{R_{\mathrm{out}}^{2}+z_{\mathrm{out}}^{2}}}\right)^{q}$,

where $\rho_{0}$ is the density of the envelope at its outer radius. We chose the mass of the envelope such that its optical depth would be the same as in the disk model. Since in this model we do not have a disk to modulate the flux of the companion, we only tested models for a $5 M_{\text {Jup }}$ central object, which provided the closest match to the companion SED. The results are shown in the middle column of Fig. 12. We find a similar match to the SED as in the disk model for a low-mass companion, but our models underestimate the degree of polarization with values at most on the order of a few $\%$ ( $\sim 7 \%$ for micron-size dust grains).

(c) Our final model is a combination of the previous two. We consider a companion surrounded by a disk plus an additional envelope. For this model, we consider a $20 M_{\text {Jup }}$ companion since it provided the best fit for the disk-only model. The density at each $(r, z)$ is taken as the maximum between $\rho_{\text {disk }}$ and $\rho_{\text {env }}$. The mass of the envelope is negligible compared to that of the disk $(\sim 0.4 \%)$. This configuration allows us to obtain a large degree of polarization by increasing the amount of scattered light with the envelope, while reducing the total intensity from the central object with an inclined disk. In the right column of Fig. 12, we show the results for three models with different grain sizes. We also varied the inclination of the disk to obtain a good fit of the data. Although none of our models fit both the photometry and level of polarization perfectly, we find that a 
disk composed of $1 \mu \mathrm{m}$ sized dust grains and a high inclination of $80^{\circ}$ is consistent with the observed photometry. This model still underpredicts the polarization in the $J$ band by a factor of 1.7. Our model using smaller grains, on the other hand, fits the HST and SPHERE $J$-band photometry slightly better, while it misses the SPHERE $H$-band and NACO $L$-band measurements. Smaller grains also lead to a dramatic overprediction of the degree of polarization in the near-infrared. Larger grains than $1 \mu \mathrm{m}$ do not significantly contribute to the degree of polarization in the $J$ and $H$ bands. Given these results it is conceivable that a more complex grain size distribution instead of a single grain size, including grain sizes between $0.1 \mu \mathrm{m}$ and $1 \mu \mathrm{m}$, may be able to reproduce the degree of polarization and the photometry. However, we would like to point out that the parameter space is complex and degenerate between multiple parameters such as companion mass, disk inclination, and dust grain size. Thus we do not claim that the disk plus dust envelope model with the given parameters is the only model that can reproduce our measurements. Additional measurements are needed before an attempt is made to constrain the nature of the companion to CS Cha further. An observation with SPHERE/ZIMPOL to detect the companion in optical polarized light could help to constrain the dust grain sizes and the presence of a dust envelope. An ALMA observation, on the other hand, may constrain the millimeter-dust mass at the companion position and thus indirectly the mass of the companion itself.

From the angle of polarization we can deduce the geometry of such a system. The angle of polarization is mostly determined by the region of the unresolved disk from which we receive the largest amount of polarized light. In the disk only model, this is the earth-facing forward scattering side of the disk, and in the disk+envelope model these are the poles of the circular envelope away from the disk. In both cases we would thus expect that the angle of polarization is aligned or closely aligned with the position angle of the circum-companion disk. We note that this scenario would change in the presence of an outflow that dominates as the source of scattered light. In such a case we would expect the angle of polarization to be perpendicular to the disk plane (Tamura \& Sato 1989). However, we have not modeled such a scenario. We have in general, not included this geometrical consideration in our models since the degree of polarization and the photometry are independent of the disk position angle.

\section{Summary and conclusions}

We observed the CS Cha system for the first time in high resolution polarimetry with SPHERE/IRDIS in $J$ and $H$ band. We resolved a circumbinary disk with an outer extent of 55.6 au in scattered light. The disk cavity predicted by previous studies was not detected owing to the limited inner working angle of the coronagraph. The upper limit for the radius of the disk cavity is $15.3 \mathrm{au}$, which is consistent with previous models by Ribas et al. (2016) using unresolved Herschel data. We find that the disk has an inclination of $24.2^{\circ} \pm 3.1^{\circ}$.

Outside of the disk at a projected separation of 214 au we find a faint companion with an extreme degree of linear polarization. To our knowledge this is the first faint and likely very low-mass companion to a nearby star that has been discovered in polarized light. With HST and NACO archival data we show with high confidence that the companion is comoving with the primary stars and is thus bound to the system, placing it at the same distance and age as CS Cha. The complex photometry of the companion could not be explained with current atmosphere models. If just the $J$ and $H$ bands were considered, a
$5 M_{\text {Jup }}$ mass may be inferred. However, this does not fit the photometry in other bands, in particular the nondetection in the $L$ band. Furthermore, a naked substellar companion is expected to have a low intrinsic polarization. Stolker et al. (2017a) showed recently that the expected degree of linear polarization from such a companion due to rotational oblateness or patchy cloud covers should not exceed $3 \%$ and is typically lower ${ }^{2}$. Thus we suggest that we are looking at a companion with a surrounding disk or dust envelope. We explored the wide parameter space for such a model with the radiative transfer code RADMC3D. We find that we can explain the companion SED and polarization reasonably well with either a highly inclined disk around a $20 M_{\text {Jup }}$ object or with a disk and additional dust envelope around an object of the same mass. This puts the companion clearly in the substellar regime: either a very low-mass brown dwarf or a high-mass planet.

From our orbit fit to the available astrometry over a time baseline of $19 \mathrm{yr}$, we can conclude that the orbit of the companion is likely eccentric with a minimum eccentricity of 0.3 . This gives some indication of how the companion may have formed. For an in situ formation, either by core accretion or by gravitational collapse in the outer circumbinary disk, one would not expect an eccentric orbit. Also the strong misalignment of the circumbinary and the circum-companion disk do not fit these scenarios. However, the eccentricity may be explained by dynamical interaction with the unresolved stellar binary. The two systems could be caught in Kozai-Lidov type resonances effectively exchanging relative inclination and eccentricity (see, e.g., Takeda \& Rasio 2005). Another possibility for an eccentric orbit would be the formation at close separations in the circumbinary disk and a subsequent dynamical scattering event in which again the central binary may have played a role. However, in such a scenario one would expect that the companion lost the surrounding disk and that some sign of perturbation would be visible in the circumbinary disk. Both do not seem to be the case.

While for typical planet formation scenarios the location and eccentricity of the orbit of the CSCha companion are problematic, this is less so for a more star-like formation by collapse in the molecular cloud in which also the CS Cha binary formed. In such a case the misaligned disks around the companion and stellar sources would also not be problematic as many such examples are known, most prominently the HK Tau system (Stapelfeldt et al. 1998), to have a similar configuration as the CS Cha system.

To better constrain the mass and properties of the companion and its surrounding disk, additional observational data is necessary, in particular, ALMA observations will allow to detect the amount of millimeter-sized dust around the companion, and likely, reveal its true nature. Additional SPHERE/ZIMPOL observations that would help to determine the grain size distribution and also potentially whether the disk or disk plus envelope scenario explains the system configuration best.

Only a few other systems are known that harbor a substellar companion with a disk around it, such as the FW Tau (Kraus et al. 2014, 2015) system or the 1SWASP J140747.93-394542.6 system (Mamajek et al. 2012; Kenworthy et al. 2015). The former is confirmed by ALMA observations, while the latter was detected in transit. However, the CS Cha system is the only system in which a resolved circumstellar disk and a circumplanetary disk are likely present. It is also to the best of our knowledge the first circumplanetary disk directly detected around a

\footnotetext{
2 See also the previous work by Sengupta \& Krishan (2001), who find
} a similar range for the degree of linear polarization. 
substellar companion in polarized light, constraining its geometry. Once the system is well understood it might be considered a benchmark system for planet and brown dwarf formation scenarios.

Acknowledgements. We thank an anonymous referee for significantly improving our original manuscript. We acknowledge I. Pascucci, M. Min M. Hogerhijde, C. Dominik, and G. Muro-Arena for interesting discussions. $\mathrm{MB}$ acknowledges funding from ANR of France under contract number ANR16-CE31-0013 (Planet Forming Disks). AJ acknowledges the support by the DISCSIM project, grant agreement 341137 funded by the European Research Council under ERC-2013-ADG. JO acknowledges support from the Universidad de Valparaíso and from ICM Núcleo Milenio de Formación Planetaria, NPF The research of FS leading to these results has received funding from the European Research Council under ERC Starting Grant agreement 678194 (FALCONER). SPHERE is an instrument designed and built by a consortium consisting of IPAG (Grenoble, France), MPIA (Heidelberg, Germany), LAM (Marseille, France), LESIA (Paris, France), Laboratoire Lagrange (Nice, France), INAF-Osservatorio di Padova (Italy), Observatoire de Geneve (Switzerland), ETH Zurich (Switzerland), NOVA (Netherlands), ONERA (France), and ASTRON (Netherlands) in collaboration with ESO. SPHERE was funded by ESO, with additional contributions from CNRS (France), MPIA (Germany), INAF (Italy), FINES (Switzerland), and NOVA (Netherlands) SPHERE also received funding from the European Commission Sixth and Seventh Framework Programmes as part of the Optical Infrared Coordination Network for Astronomy (OPTICON) under grant number RII3-Ct-2004-001566 for FP6 (2004-2008), grant number 226604 for FP7 (2009-2012) and grant number 312430 for FP7 (2013-2016). This research has made use of the SIMBAD database as well as the VizieR catalog access tool, operated at CDS, Strasbourg, France. This research has made use of NASA's Astrophysics Data System Bibliographic Services. Finally CG would like to thank Donna Keeley for language editing of the manuscript.

\section{References}

Allard, F., Hauschildt, P. H., Alexander, D. R., Tamanai, A., \& Schweitzer, A 2001, ApJ, 556, 357

ALMA Partnership, Brogan, C. L., Pérez, L. M., et al. 2015, ApJ, 808, L3

Andrews, S. M., Wilner, D. J., Zhu, Z., et al. 2016, ApJ, 820, L40

Apai, D., Pascucci, I., Wang, H., et al. 2004, IAU Symp., 221, 307 Appenzeller, I. 1977, A\&A, 61, 21

Bailey, V., Meshkat, T., Reiter, M., et al. 2014, ApJ, 780, L4

Banse, K., Crane, P., Grosbol, P., et al. 1983, The Messenger, 31, 26

Bate, M. R. 2012, MNRAS, 419, 3115

Bate, M. R. 2018, MNRAS, 475, 5618

Benisty, M., Juhasz, A., Boccaletti, A., et al. 2015, A\&A, 578, L6

Benisty, M., Stolker, T., Pohl, A., et al. 2017, A\&A, 597, A42

Bertout, C., Robichon, N., \& Arenou, F. 1999, A\&A, 352, 574

Beuzit, J.-L., Feldt, M., Dohlen, K., et al. 2008, Proc. SPIE, 7014, 18

Bonnefoy, M., Chauvin, G., Lagrange, A.-M., et al. 2014, A\&A, 562, A127

Brinch, C., Jørgensen, J. K., Hogerheijde, M. R., Nelson, R. P., \& Gressel, O. 2016, ApJ, 830, L16

Cambresy, L., Copet, E., Epchtein, N., et al. 1998, A\&A, 338, 977

Canovas, H., Ménard, F., de Boer, J., et al. 2015, A\&A, 582, L7

Canovas, H., Montesinos, B., Schreiber, M. R., et al. 2018, A\&A, 610, A13

Carbillet, M., Bendjoya, P., Abe, L., et al. 2011, Exp. Astron., 30, 39

Chabrier, G., Baraffe, I., Allard, F., \& Hauschildt, P. 2000, ApJ, 542, 464

Chauvin, G., Faherty, J., Boccaletti, A., et al. 2012, A\&A, 548, A33

Chauvin, G., Desidera, S., Lagrange, A.-M., et al. 2017, A\&A, 605, L9

Covino, E., Palazzi, E., Penprase, B. E., Schwarz, H. E., \& Terranegra, L. 1997, A\&AS, 122, 95

Cutri, R. M., Skrutskie, M. F., van Dyk, S., et al. 2003, VizieR Online Data Catalog: II/246

Cutri, R. M., Wright, E. L., Conrow, T., et al. 2012, Explanatory Supplement to the WISE All-Sky Data Release Products, Tech. rep.

Diolaiti, E., Bendinelli, O., Bonaccini, D., et al. 2000, A\&AS, 147, 335

Dohlen, K., Langlois, M., Saisse, M., et al. 2008, Proc. SPIE, 7014, 70143L

Dunham, M. M., Offner, S. S. R., Pineda, J. E., et al. 2016, ApJ, 823, 160

Espaillat, C., Calvet, N., D’Alessio, P., et al. 2007, ApJ, 664, L111

Espaillat, C., Furlan, E., D’Alessio, P., et al. 2011, ApJ, 728, 49

Esplin, T. L., Luhman, K. L., Faherty, J. K., Mamajek, E. E., \& Bochanski, J. J. 2017, AJ, 154, 46

Gauvin, L. S., \& Strom, K. M. 1992, ApJ, 385, 217

Ginski, C., Neuhäuser, R., Mugrauer, M., Schmidt, T. O. B., \& Adam, C. 2013, MNRAS, 434, 671

Ginski, C., Schmidt, T. O. B., Mugrauer, M., et al. 2014, MNRAS, 444, 2280
Ginski, C., Stolker, T., Pinilla, P., et al. 2016, A\&A, 595, A112

Girardi, L., Groenewegen, M. A. T., Hatziminaoglou, E., \& da Costa, L. 2005, A\&A, 436, 895

Girard, T. M., van Altena, W. F., Zacharias, N., et al. 2011, AJ, 142, 15

Girardi, L., Barbieri, M., Groenewegen, M. A. T., et al. 2012, Astrophys. Space Sci. Proc., 26, 165

Grady, C. A., Muto, T., Hashimoto, J., et al. 2013, ApJ, 762, 48

Guenther, E. W., Esposito, M., Mundt, R., et al. 2007, A\&A, 467, 1147

Guerri, G., Daban, J.-B., Robbe-Dubois, S., et al. 2011, Exp. Astron., 30, 59

Haisch, Jr., K. E., Lada, E. A., \& Lada, C. J. 2001, ApJ, 553, L153

Helling, C., Dehn, M., Woitke, P., \& Hauschildt, P. H. 2008, ApJ, 675, L105

Jensen-Clem, R., Millar-Blanchaer, M., Mawet, D., et al. 2016, ApJ, 820, 111

Kenworthy, M. A., Lacour, S., Kraus, A., et al. 2015, MNRAS, 446, 411

Kim, K. H., Watson, D. M., Manoj, P., et al. 2009, ApJ, 700, 1017

Kraus, A. L., Ireland, M. J., Cieza, L. A., et al. 2014, ApJ, 781, 20

Kraus, A. L., Andrews, S. M., Bowler, B. P., et al. 2015, ApJ, 798, L23

Krist, J. E., Stapelfeldt, K. R., Ménard, F., Padgett, D. L., \& Burrows, C. J. 2000, ApJ, 538, 793

Krist, J. E., Hook, R. N., \& Stoehr, F. 2011, Proc. SPIE, 8127, 81270J

Kuhn, J. R., Potter, D., \& Parise, B. 2001, ApJ, 553, L189

Langlois, M., Vigan, A., Moutou, C., et al. 2013, in Proceedings of the Third AO4ELT Conference, eds. S. Esposito, \& L. Fini, 63

Langlois, M., Dohlen, K., Vigan, A., et al. 2014, Proc. SPIE, 9147, 91471R

Lenzen, R., Hartung, M., Brandner, W., et al. 2003, Proc. SPIE, 4841, 944

Lillo-Box, J., Barrado, D., \& Bouy, H. 2012, A\&A, 546, A10

Luhman, K. L., Allen, L. E., Allen, P. R., et al. 2008, ApJ, 675, 1375

Maire, A.-L., Langlois, M., Dohlen, K., et al. 2016, Proc. SPIE, 9908, 990834

Mamajek, E. E., Quillen, A. C., Pecaut, M. J., et al. 2012, AJ, 143, 72

Manara, C. F., Testi, L., Natta, A., et al. 2014, A\&A, 568, A18

Martin, F., Conan, R., Tokovinin, A., et al. 2000, A\&AS, 144, 39

Martinez, P., Dorrer, C., Aller Carpentier, E., et al. 2009, A\&A, 495, 363

Ménard, F., Delfosse, X., \& Monin, J.-L. 2002, A\&A, 396, L35

Metchev, S. A., \& Hillenbrand, L. A. 2006, ApJ, 651, 1166

Miles-Páez, P. A., Zapatero Osorio, M. R., Pallé, E., \& Peña Ramírez, K. 2013, A\&A, 556, A125

Nguyen, D. C., Brandeker, A., van Kerkwijk, M. H., \& Jayawardhana, R. 2012, ApJ, 745, 119

Olofsson, J., van Holstein, R. G., Boccaletti, A., et al. 2018, A\&A, in press, DOI : 10. 1051/0004-6361/201832583

Pascucci, I., Ricci, L., Gorti, U., et al. 2014, ApJ, 795, 1

Pérez, L. M., Carpenter, J. M., Andrews, S. M., et al. 2016, Science, 353, 1519

Pohl, A., Sissa, E., Langlois, M., et al. 2017, A\&A, 605, A34

Pollack, J. B., Hubickyj, O., Bodenheimer, P., et al. 1996, Icarus, 124, 62

Ribas, Á., Bouy, H., Merín, B., et al. 2016, MNRAS, 458, 1029

Rousset, G., Lacombe, F., Puget, P., et al. 2003, Proc. SPIE, 4839, 140

Sauvage, J.-F., Fusco, T., Petit, C., et al. 2016, J. Astron. Telesc. Instrum. Syst., 2, 025003

Schmid, H. M., Joos, F., \& Tschan, D. 2006, A\&A, 452, 657

Schmidt, T. O. B., Neuhäuser, R., Seifahrt, A., et al. 2008, A\&A, 491, 311

Schmidt, T. O. B., Neuhäuser, R., Briceño, C., et al. 2016, A\&A, 593, A75

Sengupta, S., \& Krishan, V. 2001, ApJ, 561, L123

Serkowski, K., Mathewson, D. S., \& Ford, V. L. 1975, ApJ, 196, 261

Smart, R. L., \& Nicastro, L. 2014, A\&A, 570, A87

Stapelfeldt, K. R., Krist, J. E., Ménard, F., et al. 1998, ApJ, 502, L65

Stolker, T., Min, M., Stam, D. M., et al. 2017a, A\&A, 607, A42

Stolker, T., Sitko, M., Lazareff, B., et al. 2017b, ApJ, 849, 143

Takeda, G., \& Rasio, F. A. 2005, ApJ, 627, 1001

Tamura, M., \& Sato, S. 1989, AJ, 98, 1368

Teixeira, R., Ducourant, C., Sartori, M. J., et al. 2000, A\&A, 361, 1143

Trauger, J. T., \& WFPC2 Science Team. 1994, BAAS, 26, 892

van Boekel, R., Henning, T., Menu, J., et al. 2017, ApJ, 837, 132

van der Plas, G., Wright, C. M., Ménard, F., et al. 2017, A\&A, 597, A32

van Holstein, R. G., Snik, F., Girard, J. H., et al. 2017, Proc. SPIE, 10400 1040015

Vogt, N., Schmidt, T. O. B., Neuhäuser, R., et al. 2012, A\&A, 546, A63

Voirin, J., Manara, C. F., \& Prusti, T. 2018, A\&A, 610, A64

Wagner, K., Apai, D., Kasper, M., \& Robberto, M. 2015, ApJ, 813, L2

Walsh, C., Juhász, A., Meeus, G., et al. 2016, ApJ, 831, 200

Weingartner, J. C., \& Draine, B. T. 2001, ApJ, 548, 296

Whittet, D. C. B., Prusti, T., Franco, G. A. P., et al. 1997, A\&A, 327, 1194

Wilking, B. A., Lebofsky, M. J., \& Rieke, G. H. 1982, AJ, 87, 695

Wu, Y.-L., Close, L. M., Males, J. R., et al. 2015, ApJ, 801, 4

Yudin, R. V. 2000, A\&AS, 144, 285

Zacharias, N., Monet, D. G., Levine, S. E., et al. 2004, BAAS, 36, 1418

Zapatero Osorio, M. R., Caballero, J. A., \& Béjar, V. J. S. 2005, ApJ, 621,445 


\section{Appendix A: Stellar PSF in the SPHERE polarimetric images}

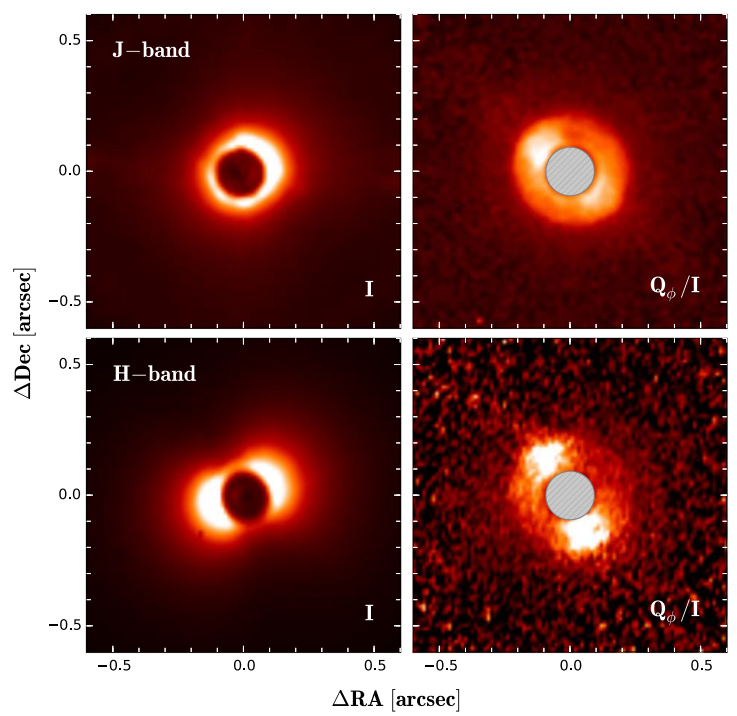

Fig. A.1. Left column: total intensity images derived from our polarimetric observations with SPHERE in the $J$ and $H$ band. The circumstellar disk is not visible in these images since flux is completely dominated by the bright stellar PSF. The visible cavity in the image center is caused by the SPHERE/IRDIS coronagraph. The stellar PSF is strongly elongated in the southeast to northwest direction in the $\mathrm{H}$ band observations. Additionally the star is not well centered behind the coronagraph, as is visible in the illumination pattern behind the (slightly transparent) coronagraph. Right column: $Q_{\phi}$ images from Fig. 1 divided by the total intensity images in the left column. This is not equivalent with polarization degree, since the intensity images are dominated by the stellar PSF rather than disk signal. The visible bright lobes are aligned with the semiminor axis of the stellar PSF, i.e., they are caused by a drop off in stellar flux and not by the polarized phase function of the disk scattered light. It is visible that the asymmetric scattered light signal in the $H$ band is less apparent in this image, indicating that it originates from the asymmetric flux in the stellar PSF which was not well centered behind the coronagraph, rather than from an actual astrophysical asymmetry in scattered light from the disk.

\section{Appendix B: Color comparison of the companion with YSOs in Cha}

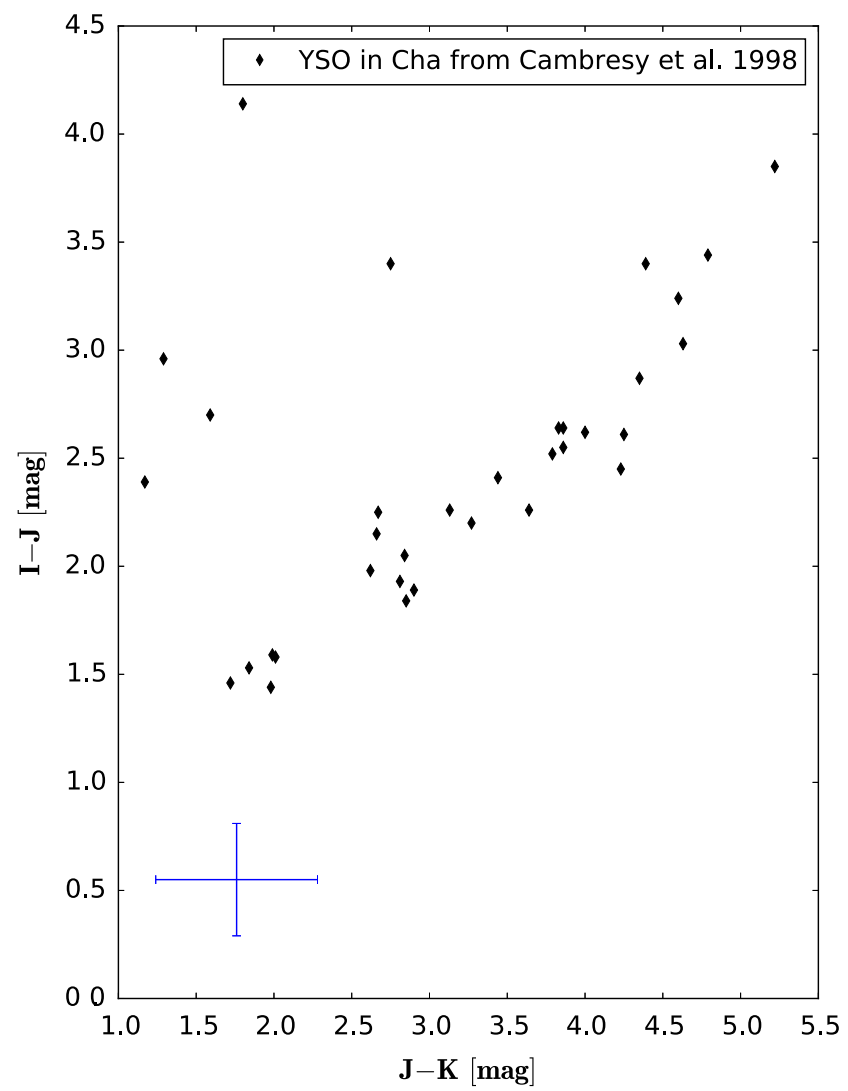

Fig. B.1. Color-color plot of YSOs in Cha from Cambresy et al. (1998). For comparison we added the companion to CS Cha in the plot as blue cross (marking the uncertainties of the photometry). The companions colors are much bluer in $I-J$ than expected for a YSO. In $J$ - $K$ they are still very blue compared to most objects. Overall we can conclude that the companion does not match YSO colors well and is in all likelihood not an embedded background YSO. 


\section{Appendix C: Model of a circum-companion disk irradiated by the central binary star}
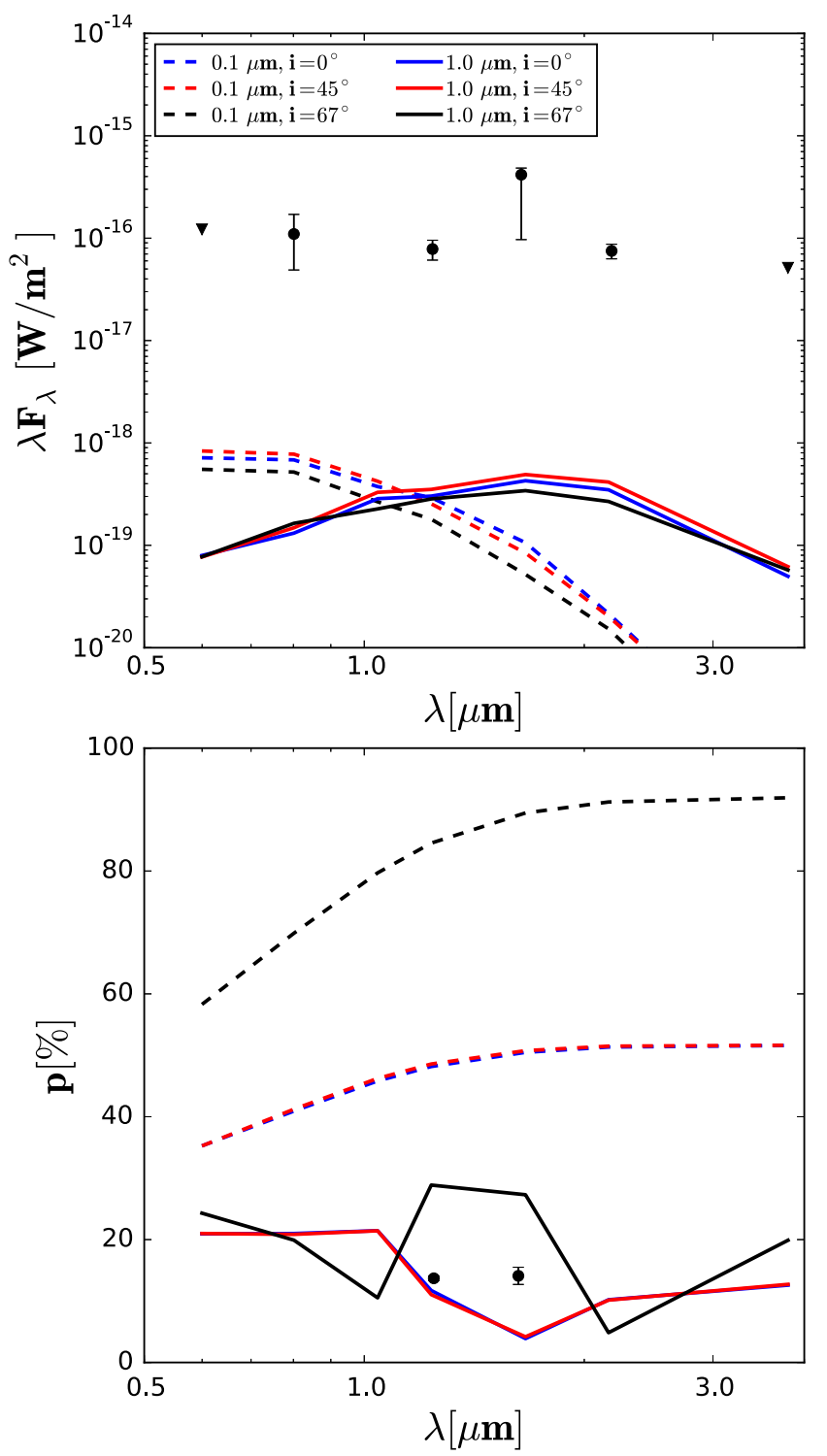

Fig. C.1. Photometry and degree of linear polarization of the companion assuming the central object is of low enough mass that its thermal emission is negligible. This very low-mass object is still surrounded by a circum-companion disk. Thus the light received from the companion is entirely scattered light from the primary stars. We used different grain sizes and inclinations of the circum-companion disk, indicated by line style and color. We find that, while we can explain the degree of linear polarization with such a model, the received flux is several orders of magnitude below our measurements. 